\title{
On the preservation of fibre direction during axisymmetric hyperelastic mass-growth of a finite fibre-reinforced tube
}

\author{
Kostas P. Soldatos
}

Received: 22 March 2017 / Accepted: 15 September 2017 / Published online: 2 December 2017

(C) The Author(s) 2017. This article is an open access publication

\begin{abstract}
Several types of tube-like fibre-reinforced tissue, including arteries and veins, different kinds of muscles, biological tubes as well as plants and trees, grow in an axially symmetric manner that preserves their own shape as well as the direction and, hence, the shape of their embedded fibres. This study considers the general, threedimensional, axisymmetric mass-growth pattern of a finite tube reinforced by a single family of fibres growing with and within the tube, and investigates the influence that the preservation of fibre direction exerts on relevant mathematical modelling, as well on the physical behaviour of the tube. Accordingly, complete sets of necessary conditions that enable such axisymmetric tube patterns to take place are initially developed, not only for fibres preserving a general direction, but also for all six particular cases in which fibres grow normal to either one or two of the cylindrical polar coordinate directions. The implied conditions are of kinematic character but independent of the constitutive behaviour of the growing tube material. Because they hold in addition to and simultaneously with standard kinematic relations and equilibrium equations, they describe growth by an overdetermined system of equations. In cases of hyperelastic mass-growth, the additional information they thus provide enables identification of specific classes of strain energy densities for growth that are admissible and, therefore, suitable for the implied type of axisymmetric tube mass-growth to take place. The presented analysis is applicable to many different particular cases of axisymmetric mass-growth of tube-like tissue, though admissible classes of relevant strain energy densities for growth are identified only for a few example applications. These consider and discuss cases of relevant hyperelastic mass-growth which (i) is of purely dilatational nature, (ii) combines dilatational and torsional deformation, (iii) enables preservation of shape and direction of helically growing fibres, as well as (iv) plane fibres growing on the cross section of an infinitely long fibre-reinforced tube. The analysis can be extended towards mass-growth modelling of tube-like tissue that contains two or more families of fibres. Potential combination of the outlined theoretical process with suitable data obtained from relevant experimental observations could lead to realistic forms of much sought strain energy functions for growth.
\end{abstract}

Keywords Anisotropic mass-growth · Axisymmetric mass-growth · Elastic-like mass-growth · Growth of cylinders and tubes $\cdot$ Hyperelastic mass-growth $\cdot$ Mass-growth modelling

K. P. Soldatos $(\varangle)$

School of Mathematical Sciences, University of Nottingham, Nottingham NG7 2RD, UK

e-mail: Kostas.Soldatos@nottingham.ac.uk

K. P. Soldatos

Spencer Institute of Theoretical and Computational Mechanics, University of Nottingham, Nottingham NG7 2RD, UK 


\section{Introduction}

Several types of tube-like hard or soft fibre-reinforced tissue possess natural ability to grow in a manner that preserves their own shape as well as the direction and, hence, the shape of their embedded fibres. Fibres of helical shape are, for instance, commonly met in several different kinds of plant and bone structures (e.g. [1-3]), as well as in various forms of tube-like soft biological tissue. The latter include arteries and veins (e.g. [4-7]), muscles (e.g. [8]) and even living creatures of tubular shape [9]. An elephant's trunk and the arm of an octopus may be referred to as additional examples of tube-like soft tissue containing families of fibres organised along several different orientations (e.g. $[10,11])$. In this context, particular mention is made of a set of experimental and theoretical investigations that aim to clarify the role of collagen fibre reinforcement as well as its influence on the mechanical behaviour of arterial wall (see [5-7] and relevant references therein). These investigations focus attention on the material anisotropy caused in different layers of the arterial vessel by the dispersion and the mean alignment of collagen fibres. It has become, for instance, understood [5] that two families of fibres are present in the intima, media and adventitia of human aortas, and these are helically arranged with respect to the cylinder axis. Often a third and sometimes a fourth fibre family is present in the intima along the respective axial and circumferential directions [5]. However, there exist also artery wall layers, such as the medial layer of human common iliac arteries, which have only a single preferred fibre direction $[6,7]$.

The present study employs postulates and principles of continuum solid mechanics with the purpose to model and subsequently investigate features associated with the ability of such tube-like types of living and growing fibrereinforced tissue to control fibre direction. Being a long established vehicle of tissue characterisation, continuum solid mechanics models tissue deformation and movement that is due to either mechanical loading (e.g. [12-18]) or mass-growth (e.g. [18-22]) on the basis of kinematical concepts and equilibrium equations that describe and balance deformation of solid media. In this context, it perceives growth of fibres that takes place naturally during mass-growth of fibre-reinforced tissue as a kind of fibre stretch.

It is important to note in this regard, that a variety of inextensible fibres embedded in growing plants, trees and, possibly, other types of living fibre-reinforced tissue resist any kind of stretch that is due to mechanical loading. However, at the same time, they naturally experience growth elongation which, in solid mechanics terms, is perceived as a kind of stretch solely due to, as well as compatible with the implied tissue growth. It follows that the concept of fibre inextensibility is generally not observable when deformation of fibre-reinforced tissue is due purely to mass-growth. More generally, there is a clear distinction between fibre extensibility or stretch due to mechanical loading and its counterpart caused by tissue mass-growth. The term "growth stretch" of a fibre is thus employed in what follows, in order to distinguish the implied fibre growth/elongation from conventional fibre stretch which is due to mechanical loading.

Under these considerations, Sect. 2 considers the most general form of axisymmetric deformation that a circular cylindrical tube may experience, and outlines preliminary details related to its kinematics and equilibrium. It also describes postulates that identify the implied deformation as a mass-growth process, quotes and discusses the boundary conditions of principal interest in this study and provides the relevant equations of dynamic equilibrium in appropriate, cylindrical polar coordinate form. Section 3 derives next conditions that should necessarily hold for a single family of fibres embedded in, and growing with the tube to preserve direction during the axisymmetric deformation of interest. In this context, fibre direction features are initially handled in a general manner. Nevertheless, a comprehensive classification is also presented of all six particular cases emerging when the fibre direction is normal to either one or two of the cylindrical polar coordinate directions.

Most of the information, arguments and concepts presented in Sects. 2 and 3 are valid regardless of whether the axially symmetric deformation of interest is due to externally applied mechanical loading or to mass-growth. Moreover, these are valid regardless of the tube material constitution, as long as the latter is consistent with the local transverse isotropy induced by the presence of a single family of fibres. In this context, Sect. 4 completes the relevant mathematical formulation by quoting basic results of a seemingly elastic-type mass-growth introduced in, hence, [22] and, hence, associating the constitutive behaviour of the growing tube with a relevant concept of "mass-growth hyperelasticity". Due to the fibre direction restrictions detailed previously in Sect. 3, the final system 
of equations that govern the described growth model may initially be perceived as overdetermined. However, the additional information provided by those restrictions can be directed towards identification of specific classes of admissible much sought strain energy densities for growth. This observation reveals then that nature can control direction of fibres that grow with and within an elastically growing tube by making use of specific forms of the corresponding strain energy density for growth.

The outlined theoretical developments are applicable to many different particular cases of axisymmetric massgrowth of tube-like tissues, some of which are considered next in Sects. 5-8. In this context, Sect. 5 introduces and discusses a particular case in which hyperelastic mass-growth is of purely dilatational nature. This discussion assists substantially the more general case detailed afterwards in Sect. 6, where dilatational and torsional mass-growth are considered combined. Thus, Sects. 5 and 6 focus on particular axisymmetric mass-growth patterns, while they both keep the fibre direction general. In contrast, Sects. 7 and 8 specify a priori the fibre shape and direction and, in better contact with practical aims of the present growth model, investigate the influence that the preservation of fibre direction exerts on both the mass-growth pattern of interest and the strain energy for growth of the tube material.

In more detail, Sect. 7 considers and discusses the aforementioned case of particular practical interest [1-11], where fibres grow with and within the tube in a helical manner. A similar kind of interest is shown afterwards for the families of plane fibres considered in Sect. 8 which, in a final application, considers cross-sectional mass growth of a transversely isotropic tube of infinite extent. It is recalled in this connection that the plane strain analysis of an infinitely long transverse isotropic tube offers some analytical simplification and is therefore not rare in conventional hyperelasticity applications (e.g. [23-28]). Useful observations stemming from the presented mass-growth modelling, analysis and applications are finally summarised in Sect. 9, which also outlines the main conclusions of this investigation as well as relevant future research directions.

\section{Preliminaries: axially symmetric deformation of a finite tube}

At $t=t_{0}$, where $t$ denotes time, an undeformed circular cylindrical tube of finite axial length, $2 H$, and constant mass density, $\rho_{0}$, occupies the region

$A \leq R \leq B, \quad 0 \leq \Theta<2 \pi, \quad-H<Z<H$,

where $R, \Theta$ and $Z$ are standard cylindrical polar coordinate parameters (Fig. 1). The non-negative constant parameters $A$ and $B(0 \leq A<B)$ represent the inner and the outer radii of the tube, respectively. If $A=0$ this representation thus depicts the particular case of a non-hollow (solid) cylinder.

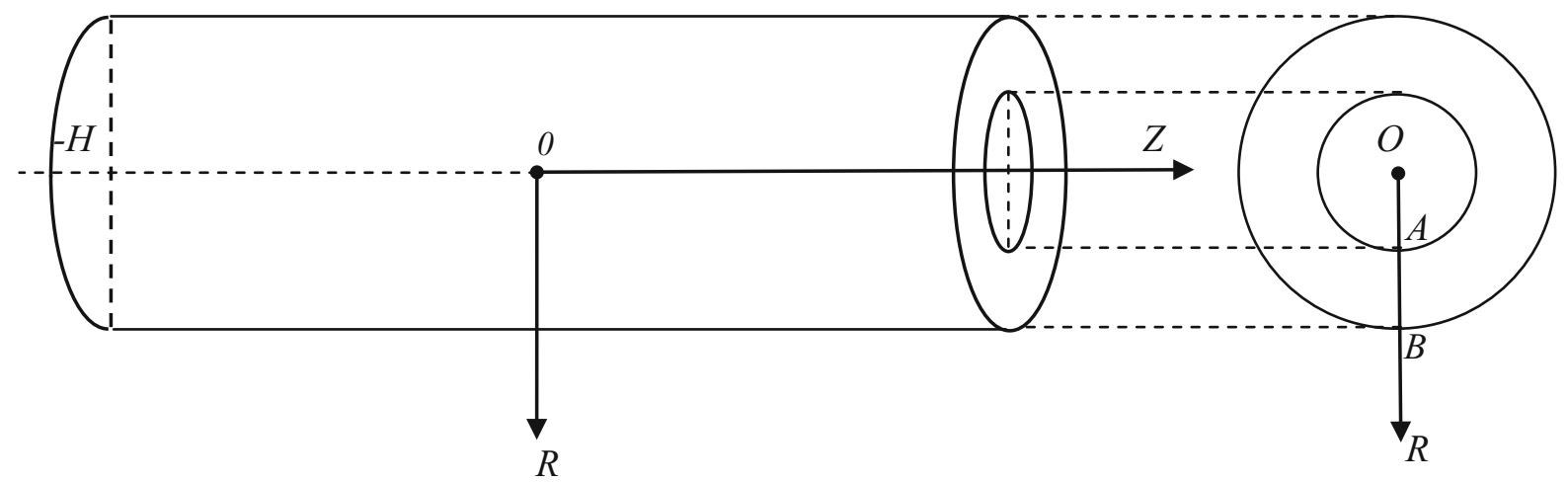

Fig. 1 Nomenclature of a finite hollow circular cylindrical tube and its cross section in the reference configuration. Nomenclature in the current configurations is obtained by replacing capital letters with their low-case version 
It is assumed that, at $t>t_{0}$, the tube deforms in a manner which is independent of the circumferential/azimuthal coordinate parameter. The most general form of such an axially symmetric deformation pattern is accordingly as follows:

$r=r(R, Z ; t), \quad \theta=\Theta+g(R, Z ; t), \quad z=z(R, Z ; t)$,

where $r, \theta$ and $z$ represent cylindrical polar coordinate parameters in the current, continuously deforming configuration. This general dynamic combination of tube radial and axial expansion with azimuthal and axial shear deformation depends on the form of the functions $r(R, Z ; t), g(R, Z ; t)=\hat{g}(r, z ; t)$ and $z(R, Z ; t)$. These are to be determined or specified, subject to the initial conditions

$r\left(R, Z ; t_{0}\right)=R, \quad g\left(R, Z ; t_{0}\right)=\hat{g}\left(r, z ; t_{0}\right)=0, \quad z\left(R, Z ; t_{0}\right)=Z$,

which preserve consistency between (2.2) and (2.1).

\subsection{Kinematics}

The basic features of the deformation pattern (2.2) are captured by the relevant deformation gradient and left Cauchy-Green deformation tensors. These are respectively described as follows:

$\boldsymbol{F}=\left[\begin{array}{ccc}\frac{\partial r}{\partial R} & \frac{\partial r}{R \partial \Theta} & \frac{\partial r}{\partial Z} \\ r \frac{\partial \theta}{\partial R} & r \frac{\partial \theta}{R \partial \Theta} & r \frac{\partial \theta}{\partial Z} \\ \frac{\partial z}{\partial R} & \frac{1}{R} \frac{\partial z}{\partial \Theta} & \frac{\partial z}{\partial Z}\end{array}\right]=\left[\begin{array}{ccc}\chi_{r} & 0 & \gamma_{\mathrm{rz}} \\ \gamma_{\theta r} & \chi_{\theta} & \gamma_{\theta \mathrm{z}} \\ \gamma_{z r} & 0 & \chi_{z}\end{array}\right]$,
$\boldsymbol{B}=\boldsymbol{F F}^{\mathbf{T}}=\left[\begin{array}{ccc}\chi_{r}^{2}+\gamma_{r z}^{2} & \gamma_{\theta r} \chi_{r}+\gamma_{\theta z} \gamma_{r z} & \chi_{r} \gamma_{z r}+\gamma_{r z} \chi_{z} \\ \gamma_{\theta r} \chi_{r}+\gamma_{\theta z} \gamma_{r z} & \gamma_{\theta r}^{2}+\chi_{\theta}^{2}+\gamma_{\theta z}^{2} & \gamma_{z r} \gamma_{\theta r}+\gamma_{\theta z} \chi_{z} \\ \chi_{r} \gamma_{z r}+\gamma_{r z} \chi_{z} & \gamma_{z r} \gamma_{\theta r}+\gamma_{\theta z} \chi_{z} & \gamma_{z r}^{2}+\chi_{z}^{2}\end{array}\right]$,

where the appearing amounts of radial, azimuthal and axial stretch are, respectively, defined according to

$\chi_{r}=r_{, R}, \quad \chi_{\theta}=r / R, \quad \chi_{z}=z_{, Z}$,

and the associated amounts of shear are

$\gamma_{r z}=r_{, Z}, \quad \gamma_{\theta r}=r g_{, R}, \quad \gamma_{\theta z}=r g_{, Z}, \quad \gamma_{z r}=z_{, R}$.

Here, as well as in what follows, a comma denotes partial differentiation with respect to the indicated coordinate parameter(s).

The radial, azimuthal and axial components of the associated velocity vector, $\boldsymbol{v}$, are

$v_{r}=\dot{r}, \quad v_{\theta}=r \dot{\theta}=r \dot{g}, \quad v_{z}=\dot{z}$,

respectively, where a dot denotes differentiation with respect to time. The assumed axial symmetry implies that the rate of deformation tensor obtains the simplified form

$\boldsymbol{d}=\left[\begin{array}{ccc}v_{r, r} & \frac{1}{2}\left(v_{\theta, r}-v_{\theta} / r\right) & \frac{1}{2} v_{z, r} \\ \frac{1}{2}\left(v_{\theta, r}-v_{\theta} / r\right) & v_{r} / r & 0 \\ \frac{1}{2} v_{z, r} & 0 & v_{z, z}\end{array}\right]=\left[\begin{array}{ccc}\dot{r}_{, r} & \frac{1}{2} r \dot{g}_{, r} & \frac{1}{2} \dot{z}_{, r} \\ \frac{1}{2} r \dot{g}_{, r} & \dot{r} / r & 0 \\ \frac{1}{2} \dot{z}_{, r} & 0 & \dot{z}_{, z}\end{array}\right]$, 
which yields further the volumetric rate of deformation as follows:

$\nabla \cdot \boldsymbol{v}=\operatorname{tr} \boldsymbol{d}=\dot{r}_{, r}+\dot{r} / r+\dot{z}_{, z}$

The deformation and equilibrium concepts outlined in this section, as well as the fibre deformation conditions detailed afterwards in Sect. 3, are independent of the cause of the assumed axisymmetric deformation. However, subsequent sections will focus on dynamic deformation patterns which are solely due to action of the rate of growth, $r_{g}$. It is accordingly fitting at this stage to introduce the rate of growth, as a scalar quantity $r_{g}$ which is a function of space and time and maintains mass-growth through its non-zero contribution in the continuity equation with growing mass,

$\dot{\rho}+\rho \nabla \cdot \boldsymbol{v}=r_{g}$,

where $\rho$ represents the mass density of the growing continuum. In the present case of interest, admissible forms of the function $r_{g}$ are evidently only those ones that enable the tube to attain and maintain axially symmetric deformation patterns of the form (2.2).

\subsection{Equilibrium}

It is assumed that stress equilibrium prevails at all times and, in the absence of body forces, is governed by the relevant quasi-static equation of motion,

$\nabla \cdot \sigma=0$

where $\sigma$ is the Cauchy stress tensor. Due to the prevailing axial symmetry considerations, the relevant cylindrical polar coordinate version of the corresponding radial, azimuthal and axial equations of motion are, respectively, simplified as follows:

$\sigma_{r r, r}+\sigma_{z r, z}+\frac{1}{r}\left(\sigma_{r r}-\sigma_{\theta \theta}\right)=0, \quad \frac{1}{r^{2}}\left(r^{2} \sigma_{r \theta}\right)_{, r}+\sigma_{z \theta, z}=0, \quad \sigma_{z z, z}+\frac{1}{r}\left(r \sigma_{r z}\right)_{, r}=0$.

The number of equations (2.12) matches the number of the principal unknown functions $r(R, Z ; t), g(R, Z ; t)$ and $z(R, Z ; t)$ appearing in (2.2). Solution of these differential equations may thus be attempted as soon as the material constitution of the tube is specified, and a relevant set of constitutive equations is thus determined; see, for instance, Sect. 4 below. Nevertheless, solution of some specific relevant boundary value problem usually requires specification of an associated set of boundary conditions.

\subsection{Boundary conditions}

It is anticipated that a deformation pattern of the form (2.2) may generally be unsustainable, unless some or all of the curved boundaries and end faces of the tube (denoted at $t>t_{0}$ with $r=a, r=b$ and $z= \pm h$ ) are supported by some set of externally applied normal tractions,

$\left.\sigma_{r r}\right|_{r=a}=q_{a}(z ; t),\left.\quad \sigma_{r r}\right|_{r=b}=q_{b}(z ; t),\left.\quad \sigma_{z z}\right|_{z= \pm h}=q_{ \pm h}(r ; t)$,

which may have to be determined in an a posteriori manner. In the same context, various conventional hyperelasticity applications (e.g. [22,28]) suggest that a presupposed axisymmetric deformation may not be sustainable without appropriate external support of the type 


$$
\begin{aligned}
& \theta-\Theta=g(A, Z ; t)=\hat{g}(a, z ; t)=\alpha(z ; t),\left.\quad \sigma_{r \theta}\right|_{r=b}=\tau_{b \theta}(z ; t), \\
&\left.\sigma_{r z}\right|_{r=a}=\tau_{a z}(z ; t),\left.\quad \sigma_{r z}\right|_{r=b}=\tau_{b z}(z ; t),\left.\quad \sigma_{z r}\right|_{z= \pm h}=\tau_{ \pm z r}(r ; t),\left.\quad \sigma_{z \theta}\right|_{z= \pm h}=\tau_{ \pm z \theta}(r ; t),
\end{aligned}
$$

which accommodates possible shear type of boundary movement.

Satisfaction of several of the non-homogeneous traction boundary conditions (2.13) and (2.14) is often required in cases where the tube deformation is due to externally applied loading. However, it will become evident in Sect. 4 below that, if the deformation pattern (2.2) is totally due to mass-growth activity, then the homogeneous version of any of those traction boundary conditions (where the corresponding $q$ - and $\tau$-quantity is zero) should be given priority and preference. If application of the non-homogeneous form of any of these boundary conditions is found necessary in subsequent sections, its presence will thus be perceived as purely supporting the observed mass-growth process.

It is also noted that, in cases that the outlined geometrical features conform to those of a corresponding nonhollow cylinder $(A=a=0)$, the boundary conditions (2.13a) and $(2.14 \mathrm{a}, \mathrm{c})$ are replaced by the requirement that the deformation and all stress components are finite on the cylinder axis $(r=0)$.

\section{Growth/deformation patterns that preserve the direction of a single family of fibres}

Consider now that the material of the tube is locally transversely isotropic due to the presence of a single family of fibres, and let the unit vector

$\boldsymbol{A}=\left\{A_{R}, A_{\Theta}, A_{Z}\right\}^{\mathrm{T}}$

denote the direction of local transverse isotropy in the reference configuration $\left(t=t_{0}\right)$. Axially symmetric deformation patterns of the form (2.2) become possible when the fibre direction vector $\boldsymbol{A}$ is generally assumed independent of the circumferential coordinate parameter. In that case, standard transformation rules of continuum mechanics reveal that, in the current tube configuration $\left(t>t_{0}\right), \boldsymbol{A}$ transforms into the vector

$\boldsymbol{b}=\left\{b_{r}, b_{\theta}, b_{z}\right\}^{\mathrm{T}}=\boldsymbol{F A}=\left\{\chi_{r} A_{R}+\gamma_{r z} A_{Z}, \gamma_{\theta r} A_{R}+\chi_{\theta} A_{\Theta}+\gamma_{\theta z} A_{Z}, \gamma_{z r} A_{R}+\chi_{z} A_{Z}\right\}^{\mathrm{T}}$,

which defines the direction of a deformed fibre.

Interest is now confined on the particular case in which the fibre direction and, therefore, the angle that it forms with any of the cylindrical polar coordinate directions remains unchanged during the axisymmetric tube growth (2.2). Upon initially assuming that

$A_{R} A_{\Theta} A_{Z} \neq 0$,

this requirement imposes on the fibre direction the restriction

$\boldsymbol{b}=\lambda(t) \boldsymbol{A}$,

or, equivalently,

$\frac{b_{r}}{A_{R}}=\frac{b_{\theta}}{A_{\Theta}}=\frac{b_{z}}{A_{Z}}=\lambda(t)$,

where $\lambda$ is the local fibre stretch or growth stretch in cases the tube deformation is purely due to mechanical loading or mass-growth, respectively. 
By eliminating $\lambda$ and using (3.2), one obtains (3.4) in the following alternative form:

$$
\begin{aligned}
& \gamma_{\theta r} A_{R}^{2}+\left(\chi_{\theta}-\chi_{r}\right) A_{R} A_{\Theta}+\left(\gamma_{\theta z} A_{R}-\gamma_{r z} A_{\Theta}\right) A_{Z}=0, \\
& \gamma_{z r} A_{R}^{2}+\left(\chi_{z}-\chi_{r}\right) A_{R} A_{Z}-\gamma_{r z} A_{Z}^{2}=0, \\
& \gamma_{\theta z} A_{Z}^{2}+\left(\chi_{\theta}-\chi_{z}\right) A_{\Theta} A_{Z}+\left(\gamma_{\theta r} A_{Z}-\gamma_{z r} A_{\Theta}\right) A_{R}=0 .
\end{aligned}
$$

Naturally, any of these restrictions can be obtained as an appropriate combination of the other two. Hence, it is sufficient for someone to require simultaneous satisfaction of any pair of (3.5).

Introduction of (2.5) and (2.6) into any two of (3.5) may then provide a pair of differential conditions which are required to hold in addition to and simultaneously with the equilibrium equations (2.12). By inserting, for instance, (2.5) and (2.6) into the first couple of (3.5), one obtains

$$
\begin{aligned}
& r g_{, R}+\left(r / R-r_{, R}\right)\left(A_{\Theta} / A_{R}\right)+\left(r g_{, Z} A_{R}-r_{, Z} A_{\Theta}\right)\left(A_{Z} / A_{R}^{2}\right)=0, \\
& z, R+\left(z, Z-r_{, R}\right)\left(A_{Z} / A_{R}\right)-r_{, Z}\left(A_{Z} / A_{R}\right)^{2}=0 .
\end{aligned}
$$

Unlike well-known classes of general material constraints that hold for all possible deformations of a material, equations (3.6) are considered applicable on the specific deformation pattern of interest, and are accordingly introduced as kinematic restrictions associated with (2.2) only. These restrictions are accordingly expected to also influence the specification process of the three unknown functions $r(R, Z ; t), g(R, Z ; t)$ and $z(R, Z ; t)$, along with the equations of motion (2.12).

The total number (five) of the governing differential equations (2.12) and (3.6) makes the mathematical model seem overdetermined. Alternatively, the additional information provided by (3.5) seems to require identification of further unknown parameters that should accompany the initial set of three unknown functions $r, g$ and $z$. Such additional unknowns may, however, be relevant to the tube material constitution or to certain privileged fibre directions. It will accordingly become more evident in Sects. 4-8 below that, in cases of hyperelastic mass-growth, (3.6) can lead to identification of privileged classes of strain energy densities that enable a tissue to control their fibre direction.

In this context, (3.6) may alternatively be regarded as a set of two simultaneous first-order linear partial differential equations, whose potential solution might lead to the elimination of two of the three unknown functions $r, g$ and $z$. The search for a general analytical solutions of (3.6) may, however, meet considerable complications, particularly in cases that the unit vector $\boldsymbol{A}$ is dependent on the cylindrical polar coordinate parameters $R$ and $Z$. Nevertheless, several classes of fibre families met often in nature violate (3.3) and, hence, lead to considerably simplified versions of (3.6) or, equivalently, (3.5). In this regard, the remaining of this section identifies all six different classes of fibre families that violate (3.3) and, for each of those classes, examines afterwards the influence that the corresponding version of (3.5) exerts on the deformation pattern (2.2).

\subsection{Classification of fibre directions that satisfy $A_{R} A_{\Theta} A_{Z}=0$}

Cases, for instance, where (i) $A_{R}=0$ or (ii) $A_{\Theta}=0$, attract immediate interest because they represent families of fibres winding on cylindrical surfaces concentric to the lateral (inner and outer) tube boundaries or growing on planes passing through the tube axis, respectively. Nevertheless, in the former case, the implied fibre families may be subdivided into three further classes, namely,

(i $A_{1} A_{R}=A_{Z}=0, A_{\Theta}=1$,

(i2) $A_{R}=A_{\Theta}=0, A_{Z}=1$,

(i3) $A_{R}=0, A_{\Theta} A_{Z} \neq 0, A_{\Theta}^{2}+A_{Z}^{2}=1$, 
while the latter case gives rise to two additional fibre classes, namely,

(ii $\left.{ }_{1}\right) A_{\Theta}=A_{Z}=0, A_{R}=1$,

(ii $) A_{\Theta}=0, A_{R} A_{Z} \neq 0, A_{R}^{2}+A_{Z}^{2}=1$.

Evidently, class $\left(i_{1}\right)$ refers to plane circumferential fibres that form concentric circles on the tube cross section while class $\left(\mathrm{i}_{2}\right)$, which might have alternatively been classified as class $\left(\mathrm{ii}_{3}\right)$, represents straight fibres aligned parallel to the tube axis. If $A_{Z}$ or, equivalently, $A_{\Theta}$ is considered constant in ( $\left.i_{3}\right)$, then that class specifies helical fibres, such as those met often in plants, arterial wall, muscle and other types of soft tissue (e.g. [1-11]). However, by considering that $A_{Z}$ may further depend on the cylindrical polar coordinate parameters, class ( $i_{3}$ ) includes also possible families of fibres that may grow in the form of irregular helices.

In a similar manner, class (ii ${ }_{1}$ ) refers to straight fibres that spread radially on the undeformed tube cross section while, if $A_{Z}$ is a non-zero constant, class (ii 2 ) specifies straight fibres passing through cylindrical axis, and forming non-zero constant angles with both that axis and the cross-sectional plane of the tube. Nevertheless, class (ii ${ }_{2}$ ) enables relevant curved plane fibres to also be considered, if $A_{Z}$ or, equivalently, $A_{R}$ depends on the cylindrical polar coordinate parameters.

The final case in which (3.3) is violated, namely,

(iii) $A_{Z}=0, \quad A_{R} A_{\Theta} \neq 0, \quad A_{R}^{2}+A_{\Theta}^{2}=1$,

refers to plane spiral fibres placed on the tube cross section. This class does not include the radial fibres considered in ( $\left.\mathrm{i}_{1}\right)$ or the circumferential fibres considered in (ii ${ }_{1}$ ) above but, evidently, includes the family of logarithmic spirals generated by any non-zero constant value of $A_{R}$ or, equivalently, $A_{\Theta}$.

3.2 Restrictions that enable preservation of fibre direction in each of the classes (i)-(iii)

It can easily be verified that (3.7a) satisfies identically all the three restrictions (3.5), thus implying that circumferential fibres always maintain their cross-sectional circumferential profile during the implied axisymmetric tube growth. Hence, in that case

(i $\left.i_{1}\right)$ no kinematic restrictions are required.

An alternative, more straightforward verification of this result is achieved by observing that, in that case, (3.2) simplifies into the following:

$\boldsymbol{b}=\left\{0, \chi_{\theta}, 0\right\}^{\mathrm{T}}$.

On the other hand, the fibre direction (3.7b) satisfies identically only the restriction (3.5a), while its connection with (3.5b) and (3.5c) reveals that straight axial fibres grow straight and remain axial only if $\gamma_{r z}=\gamma_{\theta z}=0$; thus leading to

$\left(\mathrm{i}_{2}\right) r=r(R ; t), \quad g=g(R ; t)$.

In a similar manner, connection of (3.7c) with (3.5) yields

(i 3$) r=r(R ; t), \quad z, Z=r\left(\frac{A_{Z}}{A_{\Theta}} g_{, Z}+\frac{1}{R}\right)$. 
It is observed that, for constant values of the ratio $A_{Z} / A_{\Theta},(3.12 \mathrm{~b})$ can immediately be integrated with respect to $Z$ and, hence, leads to the relationship

$z=r\left(g \frac{A_{Z}}{A_{\Theta}}+\frac{Z}{R}\right)$

This restriction is evidently consistent with the initial conditions (2.3).

Within the class $\left(\mathrm{ii}_{1}\right)$, where radial fibres are required to grow in the radial direction of the tube cross section, (3.8a) satisfies identically (3.5c). Moreover, when connected with (3.5a) and (3.5b), (3.8a) yields $\gamma_{\theta r}=\gamma_{z r}=0$, thus leading to

(ii 1$) g=g(Z ; t), \quad z=z(Z ; t)$.

In a similar manner, families of plane fibres involved in the class ( $\left.\mathrm{ii}_{2}\right)$ are found able to grow with no change of shape or direction only if

$$
\gamma_{\theta r} A_{R}+\gamma_{\theta z} A_{Z}=0, \quad \gamma_{z r} A_{R}^{2}+\left(\chi_{z}-\chi_{r}\right) A_{R} A_{Z}-\gamma_{r z} A_{Z}^{2}=0,
$$

which, with use of (2.5) and (2.6), are further converted into the following differential restrictions:

(ii $\left.i_{2}\right) g_{, R} A_{R}+g_{, Z} A_{Z}=0, \quad z, R+\left(z, Z-r_{, R}\right) A_{Z} / A_{R}-r_{, Z}\left(A_{Z} / A_{R}\right)^{2}=0$.

Finally, spiral plane fibres of the type implied in class (iii) are enabled to grow with no change of shape and direction only if

(iii) $z=z(Z ; t), \quad r g_{, R}=(r, R-r / R) \frac{A_{\Theta}}{A_{R}}$.

It is observed in this context that (3.17b) may be satisfied in one of two different ways.

Accordingly, if $g_{, R}=r, R-r / R=0$, then it is necessarily

$g=g(Z ; t), \quad r(R, Z ; t)=\phi(Z ; t) R, \quad \phi\left(Z ; t_{0}\right)=1$.

These restrictions suggest that, regardless of the non-zero value of the ratio $A_{\Theta} / A_{R}$, the tube grows radially in a linearly proportional manner that creates torsional deformation without presence of cross-sectional azimuthal shear strain; every tube cross section simply rotates about the tube axis.

The alternative manner in which (3.17b) can be satisfied relates to radially non-linear (non-proportional) tube growth of the form,

$g_{, R}=(r, R / r-1 / R) \frac{A_{\Theta}}{A_{R}}, \quad r, R-r / R \neq 0$.

If/when feasible, this creates azimuthal shear strain that depends on the value of the ratio $A_{\Theta} / A_{R}$ and, therefore, on the angle that potential spiral fibres form with the cross-sectional coordinate parameters. The special case in which the ratio $A_{\Theta} / A_{R}$ is constant becomes a case of particular interest because it includes all families of fibres shaped in the form logarithmic spirals. In that case, the first of (3.18b) is susceptible to direct integration with respect to $R$, thus leading to

$g(R, Z ; t)=\ln \{\hat{\phi}(Z ; t) r / R\}^{A_{\Theta} / A_{R}}, \quad \hat{\phi}\left(Z ; t_{0}\right)=1$. 
It is emphasised that each of the several different sets of restrictions detailed above makes possible an axisymmetric tube deformation pattern that preserves the corresponding, a priori specified fibre direction only if is also compatible with the tube equilibrium equations. Moreover, any of these sets of restrictions is applicable regardless of the type of the tube material constitution.

However, the example applications presented later in Sects. 5 and 6 depart with no restrictions on the fibre direction but, instead, impose certain simplifying assumptions on the axisymmetric deformation pattern (2.2). Sections 7 and 8 specify next a priori the fibre shape and direction and investigate the influence that the latter exerts on the corresponding axisymmetric mass-growth pattern. In this connection, Sect. 7 assumes that the fibres growing with and within the tube material are helically oriented and, hence, a priori specified as ( $\left.i_{3}\right)$-class fibres, while Sect. 8 deals with a case that may involve any of the three classes of plane cross-sectional fibres, namely $\left(\mathrm{i}_{1}\right),\left(\mathrm{ii}_{1}\right)$ or (iii). In all four of these examples, consideration of the equilibrium equations (2.12) is enabled in association with the purely hyperelastic type of mass-growth detailed next in Sect. 4.

It is finally worth recalling that in many cases of large axisymmetric tube deformation due to action of externally applied mechanical loading, the observed deformation pattern is a particular case of, and, therefore, simpler than that implied by (2.2). It is similarly very likely that several of the plethora of the different growth mechanisms met in nature can produce axisymmetric deformation patterns which are also simpler than (2.2). In such cases, several of the restriction sets obtained in this section may attain some simplified or modified form, as is demonstrated in Appendix A with an illustrative example.

\section{Mass-growth hyperelasticity}

The information, concepts and arguments presented in Sects. 2 and 3 above are valid regardless of whether the implied axially symmetric deformation is due to mass-growth or to externally applied mechanical loading, as well as regardless of the tube material constitution as long as the latter is consistent with the local transverse isotropy implied by the presence of a single family of fibres. Conventional hyperelasticity may accordingly provide the simplest possible choice of a relevant constitutive law.

However, the constitutive equation of interest in this investigation stems from its elastic-like mass-growth counterpart derived in [22] (see also Appendix B), namely,

$\boldsymbol{\sigma}=\frac{\rho}{\rho_{0}} \boldsymbol{F}\left(\frac{\partial W}{\partial \boldsymbol{C}}+\frac{\partial W}{\partial \boldsymbol{C}^{\mathrm{T}}}\right) \boldsymbol{F}^{\mathrm{T}}+\frac{r_{g}}{\rho_{0} \nabla \cdot \boldsymbol{v}} W \boldsymbol{I}$

where $W$ represents the strain energy density for growth of the tube material. This excludes the effects of possible plastic mass-growth considered in [22,29] and, for convenience, is next replaced with its simplest possible form.

It is recalled in this connection (see Sect. 1) that there is a clear distinction between fibre extensibility (or stretch) due to mechanical loading and its counterpart caused by tissue mass-growth. That distinction distinguishes the concept of "growth stretch" of a fibre from the conventional fibre stretch due to mechanical loading. This further implies that, in general, the material response mechanism that accounts for fibre growth stretch is necessarily different from its counterpart that accounts for fibre stretch due to mechanical loading. Indeed, the particular example of mechanically inextensible but otherwise normally growing fibres mentioned in the Introduction makes it obvious that the strain energy density for growth of a fibre-reinforced material is not necessarily identical with its conventional counterpart (e.g. [30]).

Alternatively, these considerations justify the claim that, in general, the strain energy density for growth of a solid tissue differs from its strain energy density due to mechanically caused deformations. In cases that mass-growth as well as some set of mechanical loads act on a solid tissue simultaneously, such a possible pair of different strain energy densities should somehow interact. However, more details of the implied kind of interaction are currently unknown, as is also unknown whether those different strain energy densities present similarities in certain types of tissue or are completely different in others. 
This investigation does not aim to explore further or answer any of the numerous questions and/or possible consequences that might follow the outlined claim. In this context, it confines interest only on axisymmetric deformation patterns which are due purely to mass-growth activity. Namely, deformation patterns of the form (2.2), which (i) are due solely to the activity of the continuity condition with growing mass (2.10), and, if/when necessary, (ii) are supported by boundary conditions that do not interfere mechanically with the implied mass-growth process. The latter restriction thus justifies the previously expressed preference on the homogeneous version of the traction boundary conditions (2.13) and (2.14).

\subsection{Incompressible mass growth. Mass-growth hyperelasticity}

In conventional hyperelasticity where $r_{g} \equiv 0$, the continuity equation with growing mass (2.10) reduces to the usual mass conservation law. The class of incompressible materials is then naturally associated with the constraint equation $\rho / \rho_{0}=1=\operatorname{det} \boldsymbol{F}$ or, equivalently, $\nabla \cdot v=0$. This is accordingly naturally identified as the class of materials which are susceptible to isochoric deformations only (e.g. [31]).

However, when mass-growth does take place $\left(r_{g} \neq 0\right)$, isochoric mass-growth converts (2.10) into

$\nabla \cdot v=0, \quad \frac{d \rho}{d t}=r_{g}$.

Hence, the concept of "isochoric mass-growth" identifies a class of kinematically constrained deformations which includes the aforementioned conventional class of incompressible continuum mechanics deformations $(\nabla \cdot v=$ $\left.r_{g} \equiv 0, \rho=\rho_{0}\right)$ as a particular case.

On the other hand, incompressible mass-growth takes place when $\rho / \rho_{0}=1 \neq \operatorname{det} \boldsymbol{F}$ and, hence, enables (2.10) to convert into the following:

$\rho=\rho_{0}, \quad \nabla \cdot \boldsymbol{v}=r_{g} / \rho_{0} \neq 0$,

Incompressible mass-growth deformation takes thus place under non-zero change of volume (det $\boldsymbol{F} \neq 1)$ and, unlike its conventional solid mechanics counterpart, is accordingly neither isochoric nor any other kind of a kinematically constrained deformation.

Looking for some reduction of the mathematical complexity involved in (4.1), one can thus consider separately possible subclasses of mass-growth deformation which are regulated by a relationship of the form

$\nabla \cdot v=s \frac{r_{g}}{\rho_{0}}$

where the non-dimensional, auxiliary parameter $s$ may either be specified a priori on the basis of existing theoretical/experimental evidence or determined a posteriori by solving some well-posed mass-growth boundary value problem. This parameter is considered independent of time, but not necessarily independent of position and, hence, not necessarily constant; see also [32].

However, the constant value $s=1$ represents all cases and classes of incompressible mass-growth (4.3), where the ratio coefficient appearing in the last term (4.1) becomes 1. Mass-growth incompressibility enables thus the general constitutive formula (4.1) to take the simpler form

$\boldsymbol{\sigma}=\boldsymbol{F}\left(\frac{\partial W}{\partial \boldsymbol{C}}+\frac{\partial W}{\partial \boldsymbol{C}^{\mathbf{T}}}\right) \boldsymbol{F}^{\mathbf{T}}+W \boldsymbol{I}$, 
which is a natural initial step of, and attracts, principal interest in the present investigation. This simplified form of (4.1) resembles closely the constitutive equation of conventional hyperelasticity and, hence, favours its association with the simplified and briefer term "mass-growth hyperelasticity".

It is emphasised that, by virtue of (4.3), incompressible mass-growth enables the mass density to remain time independent. Use of (4.3) makes thus evident that the mass-growth of present interest is possible only if the rate of growth and the deformation pattern (2.2) relate as follows:

$r_{g}=\rho_{0}\left(\dot{r}_{, r}+\dot{r} / r+\dot{z}_{, z}\right)$.

Admissible forms of the function $r_{g}$ are accordingly dictated in the present study by the form of the right-hand side of (4.6), which implies that the axially symmetric incompressible mass-growth of interest may be possible only if the rate of mass-growth is independent of the azimuthal coordinate parameter, $\theta$. It is also emphasised that $r_{g}$ influences thus directly only the dilatational part of the growth pattern (2.2). Hence, in the absence of external loading, shear amounts of the form (2.6) may be observed only in association with some kind of material anisotropy, such as the anisotropy caused by some suitably shaped preference material direction (e.g. presence of suitably curved fibres).

4.2 Mass-growth hyperelasticity for a locally transverse isotropic tube

The form of the constitutive Eq. (4.5) implies that the initial values that $W$ and $\sigma$ acquire at $t=t_{0}$ are interconnected as well as influenced by earlier mass-growth stages of the tube material [22,29]. In this regard, the following set of initial conditions should generally be associated with (4.5):

$\left.W\right|_{t=t_{0}}=W_{0},\left.\quad \boldsymbol{\sigma}\right|_{\substack{t=t_{0} \\ \boldsymbol{F}=\boldsymbol{I}}}=\left.\left(\frac{\partial W}{\partial \boldsymbol{C}}+\frac{\partial W}{\partial \boldsymbol{C}^{\mathrm{T}}}\right)\right|_{\substack{t=t_{0} \\ \boldsymbol{F}=\boldsymbol{I}}}+W_{0} \boldsymbol{I}=\boldsymbol{T}_{0}$,

where $W_{0}$ is the initial value of the strain energy density for growth and $\boldsymbol{T}_{0}$ denotes the prestress tensor. Either of these physical quantities is regarded as a known product of mass-growth activity that took place at $t \leq t_{0}$.

For the sake of simplicity, the influence of a possible prestress state that exists at $t=t_{0}$ will be disregarded in what follows, where it is accordingly assumed that

$\boldsymbol{T}_{0}=\mathbf{0}$.

Nevertheless, the manner in which a possible non-zero prestress state $\left(\boldsymbol{T}_{0} \neq \mathbf{0}\right)$ may influence the present analysis is analogous to that detailed in [22,29] for isotropic mass-growth (see also [21]).

In the usual manner, objectivity requires from the strain energy for growth, $W$, to be a function of the irreducible set of independent invariants (e.g. [30])

$I_{1}=\operatorname{tr} \boldsymbol{B}, \quad I_{2}=\frac{1}{2}\left\{(\operatorname{tr} \boldsymbol{B})^{2}-\operatorname{tr} \boldsymbol{B}^{2}\right\}, \quad I_{3}=\operatorname{det} \boldsymbol{B}, \quad I_{4}=\boldsymbol{b}^{\mathbf{T}} \boldsymbol{b}=\lambda^{2}, \quad I_{5}=\boldsymbol{b}^{\mathbf{T}} \boldsymbol{B} \boldsymbol{b}$,

where $\lambda=\sqrt{I_{4}}$ represents the fibre growth stretch introduced earlier in (3.4).

The constitutive equation (4.5) is then developed in the manner followed in conventional hyperelasticity (e.g. $[30,31])$ and, hence, leads to

$$
\begin{aligned}
\boldsymbol{\sigma} & =2\left\{\left(I_{3} W_{3}+W / 2\right) \boldsymbol{I}+\left(W_{1}+I_{1} W_{2}\right) \boldsymbol{B}-W_{2} \boldsymbol{B}^{2}+W_{4} \boldsymbol{b} \otimes \boldsymbol{b}+W_{5}(\boldsymbol{b} \otimes \boldsymbol{B} \boldsymbol{b}+\boldsymbol{B} \boldsymbol{b} \otimes \boldsymbol{b})\right\} \\
& =2\left\{\left(I_{3} W_{3}+W / 2\right) \boldsymbol{I}+W_{1} \boldsymbol{B}+W_{2}\left(I_{2} \boldsymbol{I}-I_{3} \boldsymbol{B}^{-1}\right)+W_{4} \boldsymbol{b} \otimes \boldsymbol{b}+W_{5}(\boldsymbol{b} \otimes \boldsymbol{B} \boldsymbol{b}+\boldsymbol{B} \boldsymbol{b} \otimes \boldsymbol{b})\right\},
\end{aligned}
$$


where use is made of the Cayley-Hamilton theorem, and the standard notation

$W_{n}=\frac{\partial W\left(I_{1}, I_{2}, I_{3}, I_{4}, I_{5}\right)}{\partial I_{n}} \quad(n=1,2, \ldots, 5)$

is also employed.

Connection of (4.10) with (2.4b), (2.5) and (2.6) can thus provide explicit expressions of the most general form that the constitutive equations acquire when the axisymmetric deformation pattern (2.2) of a locally transversely isotropic tube is due to incompressible, hyperelastic mass-growth. Nevertheless, further connection of (4.10) with the initial conditions (4.7) and (4.8) converts the latter into the following:

$$
\begin{aligned}
& W(3,3,1,1,1)=W_{0}, \\
& W_{1}(3,3,1,1,1)+2 W_{2}(3,3,1,1,1)+W_{3}(3,3,1,1,1)+W_{0} / 2=0, \\
& W_{4}(3,3,1,1,1)+2 W_{5}(3,3,1,1,1)=0 .
\end{aligned}
$$

These must be satisfied by any admissible form of the strain energy for growth associated with (4.10).

\section{Application 1: purely dilatational mass-growth}

Elucidation of certain special features of the outlined analysis is initially achieved by considering the special case in which the axisymmetric growth pattern of interest is purely dilatational. This is necessarily the case in which the tube grows free of shear deformation and, hence,

$\gamma_{r z}=\gamma_{\theta r}=\gamma_{\theta z}=\gamma_{z r}=0$

Connection of (5.1) with (2.6) requires from $g$ to be at most a function of time. It follows that involvement of $g$ in (2.2) represents only a rigid body rotation that includes no mass-growth deformation. One can thus incorporate $g$ into the initial polar angle parameter $\Theta$, by setting $g=0$. It is then seen that, in this case, the placement boundary condition (2.14a) necessarily acquires its homogeneous form, where $\alpha=0$.

It follows that

$\boldsymbol{F}=\operatorname{diag}\left(\chi_{r}, \chi_{\theta}, \chi_{z}\right)=\operatorname{diag}\left(r_{, R}, r / R, z, z\right), \quad \boldsymbol{B}=\operatorname{diag}\left(\chi_{r}^{2}, \chi_{\theta}^{2}, \chi_{z}^{2}\right)$

and, therefore,

$\boldsymbol{b}=\left\{b_{r}, b_{\theta}, b_{z}\right\}^{\mathrm{T}}=\left\{\chi_{r} A_{R}, \chi_{\theta} A_{\Theta}, \chi_{z} A_{Z}\right\}^{\mathrm{T}}$.

A comparison of (3.4) and (5.3) makes then evident that

$\chi_{r}=\chi_{\theta}=\chi_{z}=\lambda(t)$,

and, with subsequent use of (2.5), the axisymmetric displacement pattern (2.2) simplifies into the following:

$r=\lambda(t) R, \quad \theta=\Theta, \quad z=\lambda(t) Z ; \quad \lambda\left(t_{0}\right)=1$.

Purely dilatational tube growth that preserves fibre direction is thus, necessarily, a special case of uniform deformation. Both the radial and the axial tube dimensions grow in a linearly proportional manner. This involves a single proportionality factor, which coincides with the fibre stretch of growth, $\lambda(t)$. Validity of (5.1) and (5.4) enables then one to show that the restrictions (3.5) are all satisfied identically in this case, regardless of the fibre shape/direction. 
Alternatively, the shape and direction of a single family of fibres are always preserved within a tube that grows in the purely dilatational manner (5.5). This kind of purely dilatational mass-growth may well be met in nature. Its features are accordingly detailed and discussed in the remaining of this section, though only in the context of mass-growth incompressibility.

\subsection{Incompressible mass-growth}

Mass-growth incompressibility enables the continuity equation with growing mass to obtain the form (4.6) which, when connected with with (5.5) yields

$r_{g}=3 \rho_{0} \dot{\lambda} / \lambda$

This relationship implies that purely dilatation mass-growth is possible only when $r_{g}$ depends only on time, and its integration with respect to time yields the proportionality growth parameter as follows:

$\lambda(t)=\exp \left(\frac{1}{3 \rho_{0}} \int_{t_{0}}^{t} r_{g} \mathrm{~d} t\right)$,

where the initial condition (5.5d) is also made use of.

With no further restrictions being currently imposed on the form of $r_{g}$, one should note that the non-zero components of the velocity vector are then found to be

$\left(\begin{array}{c}v_{r} \\ v_{z}\end{array}\right)=\dot{\lambda}\left(\begin{array}{l}R \\ Z\end{array}\right)=\frac{r_{g} \exp \left(\frac{1}{3 \rho_{0}} \int_{t_{0}}^{t} r_{g} \mathrm{~d} t\right)}{3 \rho_{0}}\left(\begin{array}{l}R \\ Z\end{array}\right)$.

This expression is obtained in a manner similar to that outlined in Appendix C, which discusses briefly the case of a growth rate which is in strict adherence with the rules of quasi-static mass-growth. That discussion is, however, more relevant to the content of subsequent sections.

Validity of (5.4) allows further all expressions shown in (5.2) and (5.3) to simplify accordingly. It is thus seen that $\boldsymbol{F}$ and $\boldsymbol{B}$ depend only on time, the invariants (4.9) become

$I_{1}=3 \lambda^{2}, \quad I_{2}=3 \lambda^{4}, \quad I_{3}=\lambda^{6}, \quad I_{4}=\lambda^{2}, \quad I_{5}=3 \lambda^{4}$

and, hence, the strain energy for growth will also be dependent only on time. Explicit forms of the stress components implied in (4.10) are thus found to be as follows:

$$
\begin{aligned}
& \sigma_{r r}=2\left\{\lambda^{6} W_{3}+W / 2+\left(W_{1}+2 \lambda^{2} W_{2}\right) \lambda^{2}+\lambda^{2} A_{R}^{2}\left(W_{4}+2 \lambda^{2} W_{5}\right)\right\}, \\
& \sigma_{\theta \theta}=2\left\{\lambda^{6} W_{3}+W / 2+\left(W_{1}+2 \lambda^{2} W_{2}\right) \lambda^{2}+\lambda^{2} A_{\Theta}^{2}\left(W_{4}+2 \lambda^{2} W_{5}\right)\right\}, \\
& \sigma_{z z}=2\left\{\lambda^{6} W_{3}+W / 2+\left(W_{1}+2 \lambda^{2} W_{2}\right) \lambda^{2}+\lambda^{2} A_{Z}^{2}\left(W_{4}+2 \lambda^{2} W_{5}\right)\right\}, \\
& \sigma_{r \theta}=2 \lambda^{2} A_{R} A_{\Theta}\left(W_{4}+2 \lambda^{2} W_{5}\right), \\
& \sigma_{r z}=2 \lambda^{2} A_{R} A_{Z}\left(W_{4}+2 \lambda^{2} W_{5}\right), \\
& \sigma_{z \theta}=2 \lambda^{2} A_{\Theta} A_{Z}\left(W_{4}+2 \lambda^{2} W_{5}\right) .
\end{aligned}
$$

Unless the initial fibre direction depends on the position, these stress components and the components (5.3) of $\boldsymbol{b}$ are all independent of cylindrical polar coordinate parameters. It is then convenient for someone to distinguish 
and discuss next, separately, the case of fibre direction which is also position independent. The more general case, where purely dilatational mass-growth takes place while the unit vector $\boldsymbol{A}$ depends on position, is considered later in Sect. 5.5.

\subsection{Fibre direction independent of position}

In this case, the components $A_{R}, A_{\Theta}$ and $A_{Z}$ of the unit vector $\boldsymbol{A}$ are all constant. Hence, because $\lambda, W$ and, hence, the stress components (5.10) depend only on time, the equations of motion (2.12) simplify into the following:

$\sigma_{r r}-\sigma_{\theta \theta}=0, \quad\left(r^{2} \sigma_{r \theta}\right)_{, r}=0, \quad\left(r \sigma_{r z}\right)_{, r}=0$.

The last two of these equations can then be integrated immediately to yield

$\sigma_{r \theta}(r ; t)=\frac{b^{2} \tau_{b \theta}(t)}{r^{2}}, \quad \sigma_{r z}(r ; t)=\frac{b^{2} \tau_{b z}(t)}{r}$,

where unique identification of the arbitrary functions of time $\tau_{b \theta}$ and $\tau_{b z}$ is necessarily linked with the availability and satisfaction of the boundary conditions (2.14b) and (2.14d), respectively. The expressed priority on the homogeneous version of those boundary conditions, namely,

$\tau_{b \theta}(t)=\tau_{b z}(t)=0$,

is then found compatible with the mass-growth pattern (5.5) if

$\sigma_{r \theta}(r ; t)=\sigma_{r z}(r ; t)=0$,

throughout the growing body of the tube.

By virtue of (5.10), the equations of motion (5.11a) and (5.12) are then satisfied identically if either

$W_{4}+2 \lambda^{2} W_{5}=0$

or

$A_{R}=A_{\Theta}=0$.

Evidently, (5.16) identifies the family of axial fibres, which will be considered separately in Sect. 5.4 below.

\subsection{Non-axial fibres with direction independent of position}

In the case of non-axial fibres, (5.15) is regarded as a condition which is imposed on $W$ and, accordingly, enables partial determination of admissible forms of the strain energy for growth. In this context, (5.15) may be converted into a first-order linear partial differential equation (PDE) for $W$ in five different ways, through appropriate use of one of the following choices:

$\lambda^{2}=I_{1} / 3=\sqrt{I_{2} / 3}=\sqrt[3]{I_{3}}=I_{4} / 3=\sqrt{I_{5} / 3}$. 
For instance, the choice (5.17d) converts (5.15) into the PDE

$W_{4}+\frac{2 I_{4}}{3} W_{5}=0$

If solved with the method of the separation of variables, this provides the following class of admissible strain energy densities for growth:

$W=f_{1}\left(I_{1}, I_{2}, I_{3}\right) \exp \left\{\left(I_{5}-I_{4}^{2} / 3\right) f_{2}\left(I_{1}, I_{2}, I_{3}\right)\right\}$,

where $f_{1}$ and $f_{2}$ are arbitrary functions of the invariants $I_{1}, I_{2}$ and $I_{3}$. These functions have to be chosen in a manner that satisfies the initial conditions (4.12).

Four additional classes of admissible forms of $W$ may thus be obtained in a similar manner, by choosing $\lambda^{2}$ in a manner different from (5.17d) and, hence, converting (5.15) into a first-order PDE different than (5.18). Solution of any such a PDE will provide an additional admissible class of $W$ that, like (5.19), satisfies (5.15) and, hence, enables the constitutive equations (5.10) to attain the simplified form

$\sigma_{r r}=\sigma_{\theta \theta}=\sigma_{z z}=2\left\{\lambda^{6} W_{3}+W / 2+\left(W_{1}+2 \lambda^{2} W_{2}\right) \lambda^{2}\right\}, \quad \sigma_{r \theta}=\sigma_{r z}=\sigma_{z \theta}=0$.

This represents time evolution of a spatially uniform, equi-triaxial state of stress.

Despite the fibre-reinforced nature of the tube material, the stress state (5.20) imitates, and is essentially equivalent to the stress state that develops within a corresponding isotropic tube that grows in the proportionally dilatational manner (5.5). Accordingly, the absence of shear stress enables trivial satisfaction of the preferred, homogeneous version of the shear traction boundary conditions detailed in (2.16). However, the uniform normal stress distribution (5.20) is also present on all of the tube boundary surfaces, which need thus to be supported externally through application of the boundary normal tractions

$q_{a}(t)=q_{b}(t)=q_{ \pm h}(t)=2\left\{\lambda^{6} W_{3}+W / 2+\left(W_{1}+2 \lambda^{2} W_{2}\right) \lambda^{2}\right\}$.

Alternatively, the homogeneous version of the normal traction boundary conditions (2.13) requires

$q_{a}(t)=q_{b}(t)=q_{ \pm h}(t)=0$,

which, when compared with (5.21), leads to

$2 \lambda^{6} W_{3}+2 \lambda^{2} W_{1}+4 \lambda^{4} W_{2}+W=0$.

It follows that, if (5.23) holds simultaneously with (5.15), then the assumed dilatational growth of a fibre-reinforced tube takes place in completely stress-free manner.

Attention then naturally turns into the fact that, with appropriate use of (5.17), (5.23) is converted into one of several different versions of a first-order linear PDEs for $W$. This may be achieved in a manner similar to that in which (5.15) is earlier converted into the PDE (5.18). Solution of the pair of simultaneous first-order PDEs generated by such a conversion of (5.15) and (5.23) produces admissible forms of $W$ that enable the tube to preserve fibre shape and direction while growing in a completely stress-free manner.

For instance, appropriate use of (5.17a), (5.17b) and (5.17c) converts (5.23) into

$6 I_{3} W_{3}+2 I_{1} W_{1}+12 I_{2} W_{2}+3 W=0$ 
which is to be solved simultaneously with (5.18). Hence, introduction into (5.24) of the available solution of (5.18), namely (5.19), reveals that the functions $f_{1}$ and $f_{2}$ must satisfy the differential relationship:

$$
\begin{aligned}
& 6 I_{3}\left[f_{1,3}+\left(I_{5}-I_{4}^{2} / 3\right) f_{1} f_{2,3}\right]+2 I_{1}\left[f_{1,1}+\left(I_{5}-I_{4}^{2} / 3\right) f_{1} f_{2,1}\right] \\
& \quad+12 I_{2}\left[f_{1,2}+\left(I_{5}-I_{4}^{2} / 3\right) f_{1} f_{2,2}\right]+3 f_{1}=0 .
\end{aligned}
$$

This may be satisfied in many different ways, by essentially inserting into it arbitrary choices of either $f_{1}$ or $f_{2}$ and, subsequently, identifying the other function by solving the resulting linear PDE. During that process, which will not be pursued here any further, one has to also make sure that such an identified pair of $f_{1}$ or $f_{2}$ enables satisfaction of initial conditions (4.12) in a physically sound manner.

\subsection{Axial fibres}

In the particular case distinguished by (5.16) where the growing tube is axially reinforced, the stress components (5.10) simplify into the following:

$$
\begin{aligned}
& \sigma_{r r}=2\left\{\lambda^{6} W_{3}+W / 2+\left(W_{1}+2 \lambda^{2} W_{2}\right) \lambda^{2}\right\}, \\
& \sigma_{\theta \theta}=2\left\{\lambda^{6} W_{3}+W / 2+\left(W_{1}+2 \lambda^{2} W_{2}\right) \lambda^{2}\right\}, \\
& \sigma_{z z}=2\left\{\lambda^{6} W_{3}+W / 2+\left(W_{1}+2 \lambda^{2} W_{2}\right) \lambda^{2}+3 \lambda^{2}\left(W_{4}+2 \lambda^{2} W_{5}\right)\right\}, \\
& \sigma_{r \theta}=\sigma_{r z}=\sigma_{z \theta}=0,
\end{aligned}
$$

which still represent the time evolution of a spatially uniform state of normal stresses. However, unlike the equitriaxial stress state form (5.20), the stress state (5.26) is equi-biaxial on the tube cross section because the axial normal stress (5.26c) is generally different to its in-plane counterparts.

This stress field satisfies identically the quasi-static equations of motion (5.11a) and (5.12) when (5.13) and thus (5.14) also hold, regardless of the form of, $W$. Hence, (5.26) is a formal representation of the stress state developing within a tube with axial transverse isotropy that grows in accordance with the proportionally dilatational pattern (5.5). However, unless the strain energy for growth satisfies simultaneously both (5.15) and (5.23), the stress distribution (5.26) has to be supported by some externally applied set of appropriate boundary normal tractions.

If, for instance, the strain energy density for growth satisfies (5.23) only, then $\sigma_{r r}=\sigma_{\theta \theta}=0$ and, hence, the homogeneous version of the boundary conditions $(2.13 \mathrm{a}, \mathrm{b})$ is satisfied identically. However, the transverse normal stress is still non-zero and, hence, the top and bottom faces of the tube must be supported externally by the normal tractions

$q_{ \pm h}(t)=6 \lambda^{2}\left(W_{4}+2 \lambda^{2} W_{5}\right)$.

Alternatively, if $W$ satisfies (5.15) but violates (5.23), then (5.26) attains again the equi-triaxial form (5.20) and, hence, the tube boundaries must be supported by the set of boundary normal tractions (5.21). Nevertheless, if $W$ satisfies simultaneously both (5.15) and (5.23), then the remaining analysis and, hence, the conclusion detailed in Sect. 5.3 are still fully valid.

\subsection{Fibre direction dependent on the radial and axial coordinate parameters}

Consider finally the case in which the fibre direction vector $\boldsymbol{A}$ depends on position in such a manner that its components $A_{R}, A_{\Theta}$ and $A_{Z}$ are still independent of the circumferential coordinate parameter. Because $\boldsymbol{A}$ may depend on the radial and the axial coordinate parameters only, formation of the axially symmetric growth pattern 
(2.2) is still possible. The purely dilatational form (5.5) of mass-growth insures that $\lambda$ and $W$ remain position independent but the stress components (5.10) depend evidently now on this pair of coordinate parameters. Hence, rather than taking a simplified form, the equations of motion (2.12) remain unaltered.

Nevertheless, consideration of the following intermediate results:

$$
\begin{aligned}
\sigma_{r r, r} & =4 \lambda^{2} A_{R, R} A_{R}\left(W_{4}+2 \lambda^{2} W_{5}\right), \\
\sigma_{z r, z} & =2 \lambda^{2}\left(A_{R, Z} A_{Z}+A_{R} A_{Z, Z}\right)\left(W_{4}+2 \lambda^{2} W_{5}\right), \\
\sigma_{z z, z} & =4 \lambda^{2} A_{Z, Z} A_{Z}\left(W_{4}+2 \lambda^{2} W_{5}\right), \\
\sigma_{z \theta, z} & =2 \lambda^{2}\left(A_{\Theta, Z} A_{Z}+A_{\Theta} A_{Z, Z}\right)\left(W_{4}+2 \lambda^{2} W_{5}\right),
\end{aligned}
$$

in connection with the stress field (5.10), reveals that $\left(W_{4}+2 \lambda^{2} W_{5}\right)$ is a common factor in all terms of the equations of motion (2.12). Hence, the analysis as well as the principal results and conclusions reached earlier in this section are still valid in this case, provided that $W$ satisfies (5.15). It follows that a fibre-reinforced tube can grow in the incompressible, linearly proportional dilatational manner (5.5) and (5.7) in a completely stress-free manner and, at the same time, preserve the fibre direction, provided that the latter is independent of the circumferential coordinate parameter.

Non-dilatational mass-growth patterns are however also observed in nature, probably on a more regular basis than dilatational ones do (e.g. several types of plants and trees, hair, arteries and veins). In this regard, Sects. 6-8 connect next the analysis presented in this as well as in the preceding sections with certain axisymmetric, non-dilatational mass-growth patterns. These consider mass-growth patterns that, apart from dilatation, they also generate different kinds of shear strains.

\section{Application 2: combined dilatational and torsional mass-growth-general case $\left(A_{R} A_{\Theta} A_{Z} \neq 0\right)$}

The presence of a single family of curved fibres may superpose different kinds of shear deformations on tube dilatation and, hence, cause axisymmetric mass-growth which is more general of that considered in the preceding section. A relatively simple such mass-growth pattern is captured by specialising (2.2) as follows:

$r=r(R ; t), \quad \theta=\Theta+g(R, Z ; t), \quad z=z(Z ; t)$.

This is considered and studied in this section only for fibre directions which are independent of position.

\subsection{Kinematics}

This axisymmetric deformation mode implies that

$\gamma_{z r}=\gamma_{r z}=0$

and, because

$\boldsymbol{F}=\left[\begin{array}{ccc}\chi_{r} & 0 & 0 \\ \gamma_{\theta r} & \chi_{\theta} & \gamma_{\theta z} \\ 0 & 0 & \chi_{z}\end{array}\right]=\left[\begin{array}{ccc}r_{, R} & 0 & 0 \\ r g_{, R} & r / R & r g_{, Z} \\ 0 & 0 & z, Z\end{array}\right]$,

is a mass-growth pattern that superposes azimuthal as well as torsional shear deformation on tube dilatation. 
It follows that

$\boldsymbol{b}=\left\{b_{r}, b_{\theta}, b_{z}\right\}^{\mathrm{T}}=\left\{\chi_{r} A_{R}, \gamma_{\theta r} A_{R}+\chi_{\theta} A_{\Theta}+\gamma_{\theta z} A_{Z}, \chi_{z} A_{Z}\right\}^{\mathrm{T}}$,

and a comparison with (3.4a) or (5.3) yields

$\chi_{r}=\chi_{z}=\lambda(t), \quad \gamma_{\theta r} A_{R}+\chi_{\theta} A_{\Theta}+\gamma_{\theta z} A_{Z}=\lambda A_{\Theta}$.

With use of (2.5) and (6.2), (6.5a) yields further

$r=\lambda(t) R, \quad z=\lambda(t) Z ; \quad \lambda\left(t_{0}\right)=1$,

and, by virtue of (2.6), (6.5b), leads to the first-order linear PDE

$g_{, R} A_{R}+g_{, Z} A_{Z}=0$,

for the unknown function $g$.

Connection of (6.1) and (6.6) with the incompressible mass-growth form (4.3) of the continuity equation reveals that the mass-growth rate and the fibre growth stretch still relate according to (5.6), regardless of the potential solution of (6.7). Hence, the fibre growth stretch, $\lambda$, is still given according to (5.7) where $r_{g}$ should still depend only on time. Both (5.5) and the mass-growth pattern (6.1) with (6.6) are thus triggered by the same class of massgrowth rates and, while preserving fibre shape and direction, they lead to identical predictions of fibre growth stretch. Any of the potential mass-growth solutions sought and/or found next is thus regarded as alternative/additional to the purely dilatational ones developed in Sect. 5.

When solved with the method of the separation of variables subject to the initial condition (2.3b), (6.7) yields

$g(R, Z ; t)=\phi_{1}(t) \mathrm{e}^{\phi_{2}(t)\left(\frac{R}{A_{R}}-\frac{Z}{A_{Z}}\right)}, \quad \phi_{1}\left(t_{0}\right)=0$.

Here, $\phi_{1}$ is dimensionless and $\phi_{2}$ should have dimensions of (length $)^{-1}$ but, otherwise, these are both arbitrary functions of time. Nevertheless, (6.7) with (2.3b) admits further the simpler solution

$g(R, Z ; t)=\phi(t)\left(R A_{Z}-Z A_{R}\right), \quad \phi\left(t_{0}\right)=0$,

where the arbitrary function $\phi(t)$ should also possess dimensions of (length) ${ }^{-1}$.

It can be verified that the placement fields (6.6) and (6.9) satisfy the restrictions (3.6) when (3.3) is obeyed and, hence, the fibre direction is general. Moreover, this field satisfies the corresponding restrictions detailed in Sect. 3.2 in five of the six cases that (3.3) is violated, with the class (iii) that refers to plane, cross-sectional spiral fibres being the exception. Nevertheless, a case of cross-sectional spiral fibres that grow in the cross section of a tube of infinite extent is considered and discussed later separately in Sect. 8.3. In this regard, it is fitting to note that Sect. 7.1 is dealt later with radially proportional growth of a tube with embedded helical fibres and makes also use of the azimuthal placement expression (6.9).

Despite that (6.6) coincides with the homogeneous, pure dilatational pattern (5.5), its combination with the exponential form (6.8) of $g$ leads to a non-homogeneous form of the mass-growth pattern (6.1); this provides forms of $\boldsymbol{F}, \boldsymbol{B}$ and $W$ that depend not only on time, but also on the radial and the axial coordinate parameters. As a result, the equations of motion (2.12) cannot simplify into some form similar to (5.11).

In contrast, the simpler form of $g$ given by (6.9) is linear in both $R$ and $Z$ and, hence, its association with (6.1) and (6.6) maintains a homogeneous form of the mass-growth pattern. Moreover, this leads to 


$$
\begin{aligned}
\boldsymbol{F} & =\lambda\left[\begin{array}{ccc}
1 & 0 & 0 \\
\phi R A_{Z} & 1 & -\phi R A_{Z} \\
0 & 0 & 1
\end{array}\right], \quad \boldsymbol{B}=\lambda^{2}\left[\begin{array}{ccc}
1 & \phi R A_{Z} & 0 \\
\phi R A_{Z} & 1+\phi^{2} R^{2}\left(A_{Z}^{2}+A_{R}^{2}\right) & -\phi R A_{R} \\
0 & -\phi R A_{R} & 1
\end{array}\right], \\
\boldsymbol{B}^{-1} & =\lambda^{-2}\left[\begin{array}{ccc}
1+\left(R g_{, R}\right)^{2} & -R g_{, R} & R g_{, R} R g_{, Z} \\
-R g_{, R} & 1 & -R g_{, Z} \\
R g_{, R} R g_{, Z} & -R g, Z & 1+\left(R g_{, Z}\right)^{2}
\end{array}\right] \\
= & \lambda^{-2}\left[\begin{array}{ccc}
1+\left(\phi R A_{Z}\right)^{2} & -\phi R A_{Z} & -(\phi R)^{2} A_{R} A_{Z} \\
-\phi R A_{Z} & 1 & \phi R A_{R} \\
-(\phi R)^{2} A_{R} A_{Z} & \phi R A_{R} & 1+\left(\phi R A_{R}\right)^{2}
\end{array}\right],
\end{aligned}
$$

which are all independent of the axial coordinate parameter. The invariants (4.9) become

$$
\begin{aligned}
& I_{1}=\lambda^{2}\left[3+\phi^{2} R^{2}\left(1-A_{\Theta}^{2}\right)\right], \quad I_{2}=\lambda^{4}\left[3+\phi^{2} R^{2}\left(1-A_{\Theta}^{2}\right)\right], \\
& I_{3}=\lambda^{6}, \quad I_{4}=\lambda^{2}, \quad I_{5}=\lambda^{4}\left[1+\phi^{2} R^{2} A_{\Theta}^{2}\left(1-A_{\Theta}^{2}\right)\right],
\end{aligned}
$$

and, because constant $\boldsymbol{A}$ makes thus $W$ independent of the axial coordinate parameter, further progress is possible without excessive deviation from the mathematical analysis detailed in Sect. 5.

The form of $g$ described by (6.9) is accordingly preferred to (6.8) in the remaining of this section. It is worth noting that, with this choice of $g$, the present mass-growth pattern associates to the radial and axial components (5.8) of the velocity vector the non-zero azimuthal component

$v_{\theta}=r \dot{g}=\lambda R \dot{\phi}\left(R A_{Z}-Z A_{R}\right)=\dot{\phi}(t) R\left(R A_{Z}-Z A_{R}\right) \exp \left(\frac{1}{3 \rho_{0}} \int_{t_{0}}^{t} r_{g} \mathrm{~d} t\right)$.

In this regard, Appendix $\mathrm{C}$ proposes a possible choice of $\varphi(t)$ which is in strict adherence with the rules of quasi-static mass-growth.

\subsection{Constitutive and equilibrium equations}

Explicit forms of the stress components associated with the mass-growth pattern (6.6) and (6.9) are obtained by introducing (6.10) and (6.11) into (4.10). These are as follows:

$$
\begin{aligned}
\sigma_{r r}= & 2\left\{\lambda^{6} W_{3}+W / 2+\lambda^{4} W_{2}\left(2+\phi^{2} R^{2} A_{R}^{2}\right)+W_{1} \lambda^{2}+\lambda^{2} W_{4} A_{R}^{2}+2 \lambda^{4} W_{5} A_{R}\left(A_{R}+\phi R A_{Z} A_{\Theta}\right)\right\}, \\
\sigma_{\theta \theta}=2\left\{\lambda^{6} W_{3}+W / 2+\lambda^{4} W_{2}\left[2+\phi^{2} R^{2}\left(1-A_{\Theta}^{2}\right)\right]+\lambda^{2} W_{1}\left[1+\phi^{2} R^{2}\left(1-A_{\Theta}^{2}\right)\right]\right. & \\
& \left.+\lambda^{2} W_{4} A_{\Theta}^{2}+2 \lambda^{4} W_{5} A_{\Theta}^{2}\left[1+\phi^{2} R^{2}\left(1-A_{\Theta}^{2}\right)\right]\right\}, \\
\sigma_{z z}= & 2\left\{\lambda^{6} W_{3}+W / 2+\lambda^{4} W_{2}\left(2+\phi^{2} R^{2} A_{Z}^{2}\right)+W_{1} \lambda^{2}+\lambda^{2} W_{4} A_{Z}^{2}+2 \lambda^{4} W_{5} A_{Z}\left(A_{Z}-\phi R A_{R} A_{\Theta}\right)\right\}, \\
\sigma_{r \theta}= & 2 \lambda^{2}\left(W_{1}+\lambda^{2} W_{2}\right) \phi R A_{Z}+2 \lambda^{2} W_{4} A_{R} A_{\Theta}+2 \lambda^{4} W_{5} A_{R} A_{\Theta}\left[2+\phi R A_{Z}+\phi^{2} R^{2}\left(1-A_{\Theta}^{2}\right)\right], \\
\sigma_{r z}= & 2 \lambda^{2}\left(\lambda^{2} W_{2} \phi^{2} R^{2}+W_{4}\right) A_{R} A_{Z}+2 \lambda^{4} W_{5}\left[2 A_{R} A_{Z}+\phi R A_{\Theta}\left(A_{Z}^{2}-A_{R}^{2}\right)\right], \\
\sigma_{\theta z}= & -2 \lambda^{2}\left(W_{1}+\lambda^{2} W_{2}\right) \phi R A_{R}+2 \lambda^{2} W_{4} A_{\Theta} A_{Z}+2 \lambda^{4} W_{5} A_{\Theta}\left[2 A_{Z}-\phi R A_{R} A_{\Theta}+\phi^{2} R^{2} A_{Z}\left(1-A_{\Theta}^{2}\right)\right] .
\end{aligned}
$$


By setting $\phi=0$ and, hence, disregarding the torsional and azimuthal deformation parts of the growth pattern (6.1), all results produced so far in this section reduce naturally to their pure dilatational counterparts obtained earlier in Sect. 5. However, unlike (5.10) where all stress components depend only on time, the stress components (6.13) depend here on time as well as on the radial coordinate parameter. Nevertheless, (6.13) are still independent of the axial coordinate parameter and, because $\boldsymbol{A}$ is considered constant, the equations of motion $(2.12 \mathrm{~b}, \mathrm{c})$ still simplify into $(5.11 \mathrm{~b}, \mathrm{c})$; and, hence, still lead to (5.12).

The prioritised homogeneous version of the boundary conditions (2.14b) and (2.14d) leads thus again to (5.14) which, when connected with $(6.13 \mathrm{~d}, \mathrm{e})$, imposes on $W$ the following pair of conditions:

$$
\begin{aligned}
& \left(W_{1}+\lambda^{2} W_{2}\right) \phi R A_{Z}+W_{4} A_{R} A_{\Theta}+\lambda^{2} W_{5} A_{R} A_{\Theta}\left[2+\phi R A_{Z}+\phi^{2} R^{2}\left(1-A_{\Theta}^{2}\right)\right]=0, \\
& \left(\lambda^{2} W_{2} \phi^{2} R^{2}+W_{4}\right) A_{R} A_{Z}+\lambda^{2} W_{5}\left[2 A_{R} A_{Z}+\phi R A_{\Theta}\left(A_{Z}^{2}-A_{R}^{2}\right)\right]=0 .
\end{aligned}
$$

The radial equation of motion (2.12a) obtains now the form

$$
r \sigma_{r r, r}=\sigma_{\theta \theta}-\sigma_{r r}
$$

which, when connected with $(6.13 \mathrm{a}, \mathrm{b})$, imposes on $W$ the following additional condition:

$$
\begin{aligned}
R & \left\{\lambda^{6} W_{3}+W / 2+\lambda^{4} W_{2}\left(2+\phi^{2} R^{2} A_{R}^{2}\right)+W_{1} \lambda^{2}+\lambda^{2} W_{4} A_{R}^{2}+2 \lambda^{4} W_{5} A_{R}\left(A_{R}+\phi R A_{Z} A_{\Theta}\right)\right\}_{, R} \\
= & \lambda^{4} W_{2} \phi^{2} R^{2} A_{Z}^{2}+\lambda^{2} W_{1} \phi^{2} R^{2}\left(1-A_{\Theta}^{2}\right)+\lambda^{2} W_{4}\left(A_{\Theta}^{2}-A_{R}^{2}\right) \\
& +2 \lambda^{4} W_{5}\left[A_{\Theta}^{2}-A_{R}^{2}-\phi R A_{R} A_{Z} A_{\Theta}+\phi^{2} R^{2} A_{\Theta}^{2}\left(1-A_{\Theta}^{2}\right)\right] .
\end{aligned}
$$

Admissible forms of $W$ that preserve fibre shape and direction should accordingly satisfy simultaneously all three conditions $(6.14 \mathrm{a}, \mathrm{b})$ and $(6.16)$. Such a class of admissible forms of $W$ that is independent of the strain invariant $I_{5}$ is found in Appendix D, and is as follows:

$W\left(I_{1}, I_{2}, I_{3}, I_{4}\right)=\tilde{c}_{1} \frac{f_{1}\left(I_{1}, I_{4}\right)}{f_{3}\left(I_{1}, I_{4}\right)} \exp \left[\bar{f}\left(I_{1}, I_{4}\right)+\tilde{c}_{2} \frac{f_{3}\left(I_{1}, I_{4}\right)}{f_{1}\left(I_{1}, I_{4}\right)} I_{2}-\left(1+\tilde{c}_{2}\right) \frac{f_{3}\left(I_{1}, I_{4}\right)}{f_{2}\left(I_{1}, I_{4}\right)} I_{3}\right]+\bar{W}\left(I_{1}, I_{3}, I_{4}\right)$,

where $f_{1}, f_{2}$ and $f_{3}$ are the known functions given in (D.13). Moreover, $\tilde{c}_{1}$ and $\tilde{c}_{2}$ are arbitrary constants, while $\bar{f}\left(I_{1}, I_{4}\right)$ and $\bar{W}\left(I_{1}, I_{3}, I_{4}\right)$ are arbitrary functions of their arguments. The generality of (6.17) enables thus satisfaction of the initial conditions (4.12) in several different ways. Moreover, the process described in Appendix D may be extended towards identification of additional classes of admissible forms of $W$, which also depend on $I_{5}$ or are functions of different combinations of the strain invariants.

Substitution of such an admissible form of $W$ into (6.13) provides next corresponding explicit forms of the non-zero stress components. Alternatively, the radial normal stress is obtained in the following form:

$$
\begin{aligned}
\sigma_{r r}= & q_{a}+2 \lambda^{2} \int_{a}^{r} \frac{1}{r}\left\{\lambda^{2} W_{2} \phi^{2} R^{2} A_{Z}^{2}+W_{1} \phi^{2} R^{2}\left(1-A_{\Theta}^{2}\right)+W_{4}\left(A_{\Theta}^{2}-A_{R}^{2}\right)\right. \\
& \left.+2 \lambda^{2} W_{5}\left[A_{\Theta}^{2}-A_{R}^{2}-\phi R A_{R} A_{Z} A_{\Theta}+\phi^{2} R^{2} A_{\Theta}^{2}\left(1-A_{\Theta}^{2}\right)\right]\right\} \mathrm{d} r,
\end{aligned}
$$

after substitution of $(6.13 a, b)$ into the right-hand side of (6.15) is followed by integration in the radial direction with simultaneous use of the boundary condition (2.13a).

Connection of this result with the homogeneous version of the boundary condition (2.13b) reveals further that the outer tube boundary can still be completely free of external tractions, as long as the inner boundary is externally supported by the radial normal traction 


$$
\begin{aligned}
q_{a}= & -2 \lambda^{2} \int_{a}^{b} \frac{1}{r}\left\{\lambda^{2} W_{2} \phi^{2} R^{2} A_{Z}^{2}+W_{1} \phi^{2} R^{2}\left(1-A_{\Theta}^{2}\right)+W_{4}\left(A_{\Theta}^{2}-A_{R}^{2}\right)\right. \\
& \left.+2 \lambda^{2} W_{5}\left[A_{\Theta}^{2}-A_{R}^{2}-\phi R A_{R} A_{Z} A_{\Theta}+\phi^{2} R^{2} A_{\Theta}^{2}\left(1-A_{\Theta}^{2}\right)\right]\right\} \mathrm{d} r .
\end{aligned}
$$

Nevertheless, if the growing cylinder is non-hollow, (6.19) gives instead the value of the radial normal stress developing on the axis $(a=0)$ of the cylinder when the single-curved boundary of the latter is kept free of external tractions. Moreover, and, regardless of whether the growing cylinder is hollow or not, a combination of (6.18) with (6.15) yields the hoop stress in the following alternative form:

$$
\begin{aligned}
\sigma_{\theta \theta}= & q_{a}+2 \lambda^{2} \int_{a}^{r} \frac{1}{r}\left\{\lambda^{2} W_{2} \phi^{2} R^{2} A_{Z}^{2}+W_{1} \phi^{2} R^{2}\left(1-A_{\Theta}^{2}\right)+W_{4}\left(A_{\Theta}^{2}-A_{R}^{2}\right)\right. \\
& \left.+2 \lambda^{2} W_{5}\left[A_{\Theta}^{2}-A_{R}^{2}-\phi R A_{R} A_{Z} A_{\Theta}+\phi^{2} R^{2} A_{\Theta}^{2}\left(1-A_{\Theta}^{2}\right)\right]\right\} \mathrm{d} r+2 \lambda^{2}\left\{\lambda^{2} W_{2} \phi^{2} R^{2} A_{Z}^{2}\right. \\
& \left.+W_{1} \phi^{2} R^{2}\left(1-A_{\Theta}^{2}\right)+W_{4}\left(A_{\Theta}^{2}-A_{R}^{2}\right)+2 \lambda^{2} W_{5}\left[A_{\Theta}^{2}-A_{R}^{2}-\phi R A_{R} A_{Z} A_{\Theta}+\phi^{2} R^{2} A_{\Theta}^{2}\left(1-A_{\Theta}^{2}\right)\right]\right\} .
\end{aligned}
$$

The stress components $\sigma_{z z}$ and $\sigma_{z \theta}$ will evidently still be given by (6.13c) and (6.13f), respectively. These are generally not expected to satisfy the homogeneous version of the boundary conditions (2.13c) or (2.14f), respectively. The outlined axisymmetric mass-growth process will then be possible only if the externally applied boundary tractions $q_{ \pm h}$ and $\tau_{ \pm z \theta}$ are chosen to balance the values that $\sigma_{z z}$ and $\sigma_{z \theta}$ attain on the top and bottom boundaries of the tube.

\section{Application 3: radially proportional growth of a tube with embedded helical fibres $\left(A_{R}=0\right)$}

Consider now axisymmetric mass-growth of a tube that preserves the direction of an embedded family of helical fibres. This is the class of fibres denominated as $\left(i_{3}\right)$ in Sect. 3 and, hence, initiates a search for possible axially symmetric mass-growth patterns that comply with the conditions (3.12). For the sake of relative simplicity, this search embraces the rule of proportional radial growth,

$r=\lambda(t) R ; \quad \lambda\left(t_{0}\right)=1$,

which has been previously employed in (5.5) and (6.6), and satisfies (3.12a).

Because

$A_{R}=0$

the analysis detailed in the preceding section is generally not applicable in this example. Nevertheless, with both $A_{\Theta}$ and $A_{Z}$ being non-zero constants, combination of (3.12b) with (7.1) leads to

$z(R, Z ; t)=\lambda(t)\left[Z+\frac{A_{Z}}{A_{\Theta}} R g(R, Z ; t)\right]$,

which holds between $z$ and $g$. Hence, use of (2.5) and (2.6) yields further

$$
\begin{aligned}
& \chi_{r}=\chi_{\theta}=\lambda, \quad \chi_{z}=\lambda+\gamma_{\theta z} A_{Z} / A_{\Theta}=\lambda\left(1+R g_{, Z} A_{Z} / A_{\Theta}\right), \\
& \gamma_{r z}=0, \quad \gamma_{\theta r}=\lambda R g_{, R}, \quad \gamma_{\theta z}=\lambda R g_{, Z}, \quad \gamma_{z r}=\lambda\left(g+R g_{, R}\right) A_{Z} / A_{\Theta} .
\end{aligned}
$$

The fact that only one placement component, say $g$, may now be considered unknown implies that this may be determined with use of one of the equations of motion (2.12). The remaining pair of those equations may then be 
used towards identification of relevant classes of admissible strain energy densities. However, $g$ is generally now a function of both $R$ and $Z$ and, hence, the equations of motion (2.12) cannot attain unconditionally their simplified version $(5.14 \mathrm{~b}, \mathrm{c})$ and $(6.15)$. The experience gained in previous sections suggests however that the analysis may simplify if the form of the last remaining placement component is provided. In such a case, admissible forms of $W$ should satisfy all three equations (2.12).

7.1 Kinematics associated with a particular form of the azimuthal placement component

For instance, a combination of (6.9) with (7.2) and (7.3) yields

$g=\phi(t) R A_{Z}, \quad z=\lambda(t)\left[Z+\phi(t) A_{Z}^{2} R^{2} / A_{\Theta}\right] ; \quad \phi\left(t_{0}\right)=0$,

where $\phi(t)$ may still be considered as an arbitrary function that possesses dimensions of (length) $)^{-1}$. The axisymmetric growth patterns (7.1) and (7.5) exhibit thus a specific theoretical connection with its counterpart considered in the preceding section, while its linearity with respect to the axial tube parameter enables the equations of motion (2.12) to attain again their simplified form $(5.11 \mathrm{~b}, \mathrm{c})$ and $(6.15)$.

However, the form of the axial placement (7.5b) is quadratic in the radial coordinate parameter and, hence, the placement fields (7.1) and (7.5) do not anymore represent a homogeneous deformation. Moreover, unlike either (5.5) or (6.6) with (6.9) which predict that $(b-a) /(B-A)=h / H$, the present deformation rule enables consideration of growth patterns which, as often happens in nature, do not preserve the ratio of the radial and axial tube dimensions. This is because the azimuthal placement (7.5a) influences now the magnitude of its axial counterpart (7.5b).

Use of (2.7) yields thus the components of the velocity vector as follows:

$$
\begin{aligned}
v_{r} & =\dot{\lambda} R=\frac{\lambda r_{g} R}{3 \rho_{0}}=\frac{r_{g} R}{3 \rho_{0}} \exp \left(\frac{1}{3 \rho_{0}} \int_{t_{0}}^{t} r_{g} \mathrm{~d} t\right), \\
v_{\theta} & =r \dot{\theta}=r \dot{g}=\dot{\phi}(t) R^{2} A_{Z} \exp \left(\frac{1}{3 \rho_{0}} \int_{t_{0}}^{t} r_{g} \mathrm{~d} t\right), \\
v_{z} & =\dot{\lambda} Z+(\dot{\lambda} \phi+\lambda \dot{\phi}) A_{Z}^{2} R^{2} / A_{\Theta}=\frac{\lambda r_{g}}{3 \rho_{0}} Z+\lambda\left(\frac{r_{g}}{3 \rho_{0}} \phi+\dot{\phi}\right) A_{Z}^{2} R^{2} / A_{\Theta} \\
& =\left\{\frac{r_{g}}{3 \rho_{0}} Z+\left(\frac{r_{g}}{3 \rho_{0}} \phi+\dot{\phi}\right) A_{Z}^{2} R^{2} / A_{\Theta}\right\} \exp \left(\frac{1}{3 \rho_{0}} \int_{t_{0}}^{t} r_{g} \mathrm{~d} t\right),
\end{aligned}
$$

where use is also made of the fact that (5.6) and, therefore, (5.7) still hold in the present application. The functions $\lambda(t)$ and $\phi(t)$ may thus still be given according to (C.1) and (C.6), respectively, although the product $\dot{\lambda} \phi$ appearing in (7.6c) prevents now a strict adherence with the concept of quasi-static mass-growth.

Under these considerations, the relationships (7.4) simplify as follows:

$$
\begin{aligned}
& \chi_{r}=\chi_{\theta}=\chi_{z}=\lambda, \\
& \gamma_{r z}=\gamma_{\theta z}=0, \quad \gamma_{\theta r}=\lambda \phi R A_{Z}, \quad \gamma_{z r}=2 \lambda \phi R A_{Z}^{2} / A_{\Theta},
\end{aligned}
$$

making thus more evident that the fibre growth stretch, $\lambda$, is still given according to (5.7). Any of the potential mass-growth solutions sought in what follows may thus still be regarded as additional to those obtained previously in Sects. 5 and 6, although, here, are relevant only to growth patterns of a tube reinforced by helical fibres.

Connection of (7.7) with (2.4) yields next

$$
\begin{aligned}
& \boldsymbol{F}=\lambda\left[\begin{array}{ccc}
1 & 0 & 0 \\
\phi R A_{Z} & 1 & 0 \\
2 \phi R A_{Z}^{2} / A_{\Theta} & 0 & 1
\end{array}\right], \quad \boldsymbol{B}=\boldsymbol{F} \boldsymbol{F}^{\mathrm{T}}=\lambda^{2}\left[\begin{array}{ccc}
1 & \phi R A_{Z} & 2 \phi R A_{Z}^{2} / A_{\Theta} \\
\phi R A_{Z} & 1+\phi^{2} R^{2} A_{Z}^{2} & 2 \phi^{2} R^{2} A_{Z}^{3} / A_{\Theta} \\
2 \phi R A_{Z}^{2} / A_{\Theta} & 2 \phi^{2} R^{2} A_{Z}^{3} / A_{\Theta} & 1+4 \phi^{2} R^{2} A_{Z}^{4} / A_{\Theta}^{2}
\end{array}\right], \\
& \boldsymbol{B}^{-1}=\boldsymbol{F}^{-\mathrm{T}} \boldsymbol{F}^{-1}=\lambda^{-2}\left[\begin{array}{ccc}
1+\phi^{2} R^{2} A_{Z}^{2}\left(1+4 A_{Z}^{2} / A_{\Theta}^{2}\right) & -\phi R A_{Z} & -2 \phi R A_{Z}^{2} / A_{\Theta} \\
-\phi R A_{Z} & 1 & 0 \\
-2 \phi R A_{Z}^{2} / A_{\Theta} & 0 & 1
\end{array}\right],
\end{aligned}
$$


and, consequently, enables one to obtain the invariants (4.9) as follows:

$$
\begin{aligned}
& I_{1}=\lambda^{2}\left[3+\phi^{2} R^{2} A_{Z}^{2}\left(1+4 A_{Z}^{2} / A_{\Theta}^{2}\right)\right], \quad I_{2}=\lambda^{4}\left[3+\phi^{2} R^{2} A_{Z}^{2}\left(1+4 A_{Z}^{2} / A_{\Theta}^{2}\right)\right], \\
& I_{3}=\lambda^{6}, \quad I_{4}=\lambda^{2}, \quad I_{5}=\lambda^{4}\left[1+\phi^{2} R^{2} A_{Z}^{2}\left(1+3 A_{Z}^{2}+4 A_{Z}^{4} / A_{\Theta}^{2}\right)\right] .
\end{aligned}
$$

These are all independent of the axial coordinate of the tube and, hence, the strain energy for growth will be also independent of that coordinate. The analysis can thus follow the steps detailed in the preceding section.

\subsection{Constitutive and equilibrium equations}

By inserting (7.8) and (7.9) into (4.10), the stress components are thus obtained explicitly as follows:

$$
\begin{aligned}
\sigma_{r r}= & 2\left\{\lambda^{6} W_{3}+W / 2+2 \lambda^{4} W_{2}+W_{1} \lambda^{2}\right\}, \\
\sigma_{\theta \theta}= & 2\left\{\lambda^{6} W_{3}+W / 2+\lambda^{4} W_{2}\left[2+\phi^{2} R^{2} A_{Z}^{2}\left(1+4 A_{Z}^{2} / A_{\Theta}^{2}\right)\right]+\lambda^{2} W_{1}\left(1+\phi^{2} R^{2} A_{Z}^{2}\right)\right. \\
& \left.+\lambda^{2} W_{4} A_{\Theta}^{2}+2 \lambda^{4} W_{5}\left[A_{\Theta}^{2}+\phi^{2} R^{2} A_{Z}^{2}\left(1+A_{Z}^{2}\right)\right]\right\}, \\
\sigma_{z z}= & 2\left\{\lambda^{6} W_{3}+W / 2+\lambda^{4} W_{2}\left[2+\phi^{2} R^{2} A_{Z}^{2}\left(1+4 A_{Z}^{2} / A_{\Theta}^{2}\right)\right]+\lambda^{2} W_{1}\left(1+4 \phi^{2} R^{2} A_{Z}^{4} / A_{\Theta}^{2}\right)\right. \\
& \left.+\lambda^{2} W_{4} A_{Z}^{2}+2 \lambda^{4} W_{5} A_{Z}^{2}\left[1+2 \phi^{2} R^{2} A_{Z}^{2}\left(1+2 A_{Z}^{2} / A_{\Theta}^{2}\right)\right]\right\}, \\
\sigma_{r \theta}= & 2 \lambda^{2} \phi R A_{Z}\left[W_{1}+\lambda^{2} W_{2}+\lambda^{2} W_{5}\left(1+A_{Z}^{2}\right)\right], \\
\sigma_{r z}= & 2 \lambda^{2} \phi R A_{Z}\left[2\left(W_{1}+\lambda^{2} W_{2}\right)+\lambda^{2} W_{5}\left(1+A_{Z}^{2}\right)\right] A_{Z} / A_{\Theta}, \\
\sigma_{\theta z}= & 2 \lambda^{2}\left\{\phi^{2} R^{2} A_{Z}^{2}\left[2 W_{1}+3 \lambda^{2} W_{5}\left(1+A_{Z}^{2}\right)\right]+\left(W_{4}+2 \lambda^{2} W_{5}\right) A_{\Theta}^{2}\right\} A_{Z} / A_{\Theta} .
\end{aligned}
$$

By setting $\phi=0$ and, hence, restricting attention on the corresponding purely dilatational mass-growth discussed in Sect. 5, (7.10) reduce naturally into the form attained by (5.10) when (7.2) is also taken into consideration. This observation is a confirmation of the fact that purely dilatational mass-growth of a tube that preserves the direction of an embedded family of helical fibres is indeed possible when the strain energy density for growth satisfies the differential condition (5.18). However, that condition is violated in the present case of interest, where possible forms of $W$ that enable realisation of the mass-growth pattern (7.1) and (7.5) should necessarily obey some set of different restrictions.

Those restrictions are obtained by inserting (7.10) into the simplified versions (5.14) and (6.15) of the equations of motion which, as already mentioned, still hold. They are accordingly as follows:

$$
\begin{aligned}
W_{1}+\lambda^{2} W_{2}+\lambda^{2} W_{5}\left(1+A_{Z}^{2}\right)=0, & \\
2\left(W_{1}+\lambda^{2} W_{2}\right)+\lambda^{2} W_{5}\left(1+A_{Z}^{2}\right)= & 0, \\
R\left\{\lambda^{4} W_{3}+W / 2+2 \lambda^{2} W_{2}+W_{1}\right\}_{, R}= & \phi^{2} R^{2} A_{Z}^{2}\left[W_{1}+\lambda^{2} W_{2}\left(1+4 A_{Z}^{2} / A_{\Theta}^{2}\right)+2 \lambda^{2} W_{5}\left(1+A_{Z}^{2}\right)\right] \\
& +\left(W_{4}+2 \lambda^{2} W_{5}\right) A_{\Theta}^{2} .
\end{aligned}
$$

The first two of (7.11) can hold simultaneously only if

$W_{1}+\lambda^{2} W_{2}=W_{5}=0$.

Appendix E shows that (7.11c) can then simplify, and become

$W_{4}+4 \lambda^{2} \phi^{2} R^{2}\left(A_{Z} / A_{\Theta}\right)^{2} W_{2}=0$. 
Potential forms of $W$ that satisfy simultaneously all three differential conditions (7.12) and (7.13) may be sought in the manner described in the preceding sections. Nevertheless, the form

$W=W\left(I_{3}\right)$

is considered as the simplest relevant choice. Because $I_{3}^{1 / 2}$ is a measure of volume change, this fits also well the dilatational features of the incompressible mass-growth of interest; see also (4.2), (5.6) and (5.7).

It is then observed with interest that connection of (7.14) with (7.10) produces the equi-triaxial stress field

$\sigma_{r r}=\sigma_{\theta \theta}=\sigma_{z z}=2\left(\lambda^{6} W_{3}+W / 2\right)=2\left(I_{3} W_{3}+W / 2\right)$,

$\sigma_{r \theta}=\sigma_{r z}=\sigma_{\theta z}=0$,

which neither depends on the azimuthal nor on the torsional amounts of shear encountered in (7.7). This observation implies further that neither the shear parts of this mass-growth deformation nor the speed that they evolve through time influence the observed equi-triaxial stress field.

The stress field (7.15) seems then associated with an essentially infinite number of mass-growth patterns of the form (7.1) and (7.5), and this concept of placement arbitrariness is manifested in (7.5) through the involvement of the function $\phi(t)$. Each of those patterns is caused by the same evolution rule of dilatational growth, represented in (7.15) by a certain choice of the function $\lambda(t)$, but involves some different rules of shear strain growth, due to the different possible choices of the function $\phi(t)$. The choice of this pair of otherwise arbitrary functions dictates thus substantially the manner in which shear deformation that is caused through growth of unidirectional helical fibres alters the proportional, single-parameter features of the mass-growth patterns (5.5) and (6.6) considered in Sects. 5 and 6, respectively. The form of (7.5) then reveals that some specific link, similar to that described in Appendix C, may need to be sought and found between $\lambda(t)$ and $\phi(t)$.

The kind of axisymmetric mass-growth encountered in this section for a tube reinforced by a single family of helical fibres is evidently possible only if the equi-triaxial stress field (7.15) is supported by appropriate normal tractions that act externally on all the curved and flat boundaries of the tube. It is finally noted that a simple polynomial example of (7.14) that satisfies the initial conditions (4.13) is as follows:

$W=\left(\frac{n+1 / 2}{n-m} I_{3}^{m}-\frac{m+1 / 2}{n-m} I_{3}^{n}\right) W_{0}$,

where $m$ and $n$ represent unequal positive integers $(m \neq n)$; the corresponding equi-triaxial stress field may then easily be calculated with use of (7.15).

\section{Application 4: Growth of an infinitely long tube with an embedded family of spiral fibres}

This final application considers the particular case of two-dimensional, plane strain mass-growth that takes place on the cross section of a transverse isotropic tube of infinite extent. Accordingly, the reference configuration (2.1) of the tube growth is described as follows:

$A \leq R \leq B, \quad 0 \leq \Theta<2 \pi, \quad-\infty<Z<\infty$,

and, because mass-growth is independent of the axial coordinate parameter, the general axisymmetric deformation pattern (2.2) takes the simplified, two-dimensional form

$r=r(R ; t), \quad \theta=\Theta+g(R ; t), \quad z=Z$. 
All vectors and tensors appearing in this section are accordingly considered two-dimensional, the stress tensor being the only exception. This is because a two-dimensional version of (4.5) needs to be complemented by the out-of-plane normal stress component, which is in general non-zero. For the sake of simplicity though, attention in what follows is confined on the in-plane part of the stress state only.

It follows that only three of the seven gradient components (2.5) and (2.6) are still non-zero, namely

$\chi_{r}=r_{, R}, \quad \chi_{\theta}=r / R, \quad \gamma \equiv \gamma_{\theta r}=r g_{, R}$.

Moreover, the $3 \times 3$ tensors defined in Sect. 2 attain here the following two-dimensional forms:

$$
\begin{aligned}
& \hat{\boldsymbol{F}}=\left[\begin{array}{cc}
\chi_{r} & 0 \\
\gamma & \chi_{\theta}
\end{array}\right], \quad \hat{\boldsymbol{B}}=\hat{\boldsymbol{F}} \hat{\boldsymbol{F}}^{\mathrm{T}}=\left[\begin{array}{cc}
\chi_{r}^{2} & \gamma \chi_{r} \\
\gamma \chi_{r} & \gamma^{2}+\chi_{\theta}^{2}
\end{array}\right], \\
& \hat{\boldsymbol{d}}=\left[\begin{array}{cc}
v_{r, r} & \frac{1}{2}\left(v_{\theta, r}-v_{\theta} / r\right) \\
\frac{1}{2}\left(v_{\theta, r}-v_{\theta} / r\right) & v_{r} / r
\end{array}\right]=\left[\begin{array}{cc}
\dot{r}_{, r} & \frac{1}{2} r \dot{g}_{, r} \\
\frac{1}{2} r \dot{g}_{, r} & \dot{r} / r
\end{array}\right],
\end{aligned}
$$

and the relation (4.6) between the rate of growth and the deformation pattern simplifies into the following:

$r_{g}=\rho_{0}\left(\dot{r}_{, r}+\dot{r} / r\right)$.

Moreover, because $A_{Z}=b_{z}=0$, the relationship (3.2) obtains the simpler form

$\hat{\boldsymbol{b}}=\left\{b_{r}, b_{\theta}\right\}^{\mathrm{T}}=\hat{\boldsymbol{F}} \hat{\boldsymbol{A}}=\left\{\chi_{r} A_{R}, \gamma A_{R}+\chi_{\theta} A_{\Theta}\right\}^{\mathrm{T}}, \quad \hat{\boldsymbol{A}}=\left\{A_{R}, A_{\Theta}\right\}^{\mathrm{T}}$.

It becomes also evident that, with $A_{Z}=0$, classes of fibre families which are of interest in this application, namely those denoted in Sect. 3 as $\left(i_{1}\right),\left(i i_{1}\right)$ and (iii), are formed by circumferential, axial and spiral fibres, respectively. Those three families are accordingly considered separately in what follows.

The reduction of spatial dimensions makes the strain invariants $I_{3}$ and $I_{5}$ redundant (e.g. [30]), and the remaining independent invariants simplify as follows:

$$
\begin{aligned}
& J_{1} \equiv I_{1}=\operatorname{tr} \hat{\boldsymbol{C}}=\operatorname{tr} \hat{\boldsymbol{B}}=\chi_{r}^{2}+\gamma^{2}+\chi_{\theta}^{2}, \\
& J_{2} \equiv I_{2}=\operatorname{det} \hat{\boldsymbol{C}}=\operatorname{det} \hat{\boldsymbol{B}}=\chi_{r}^{2} \chi_{\theta}^{2}, \\
& J_{3} \equiv I_{4}=\hat{\boldsymbol{A}}^{\mathrm{T}} \hat{\boldsymbol{C}} \hat{\boldsymbol{A}}=\hat{\boldsymbol{b}}^{\mathrm{T}} \hat{\boldsymbol{b}}=\lambda^{2}=\left(\chi_{r}^{2}+\gamma^{2}\right) A_{R}^{2}+\chi_{\theta}^{2} A_{\Theta}^{2}+2 \gamma \chi_{\theta} A_{R} A_{\Theta} .
\end{aligned}
$$

The two-dimensional version of the constitutive equation (4.10) is thus represented as follows:

$\sigma=2\left\{\left(\hat{W}_{1}+J_{1} \hat{W}_{2}\right) \hat{\boldsymbol{B}}-\hat{W}_{2} \hat{\boldsymbol{B}}^{2}+\hat{W}_{3} \hat{\boldsymbol{b}} \otimes \hat{\boldsymbol{b}}\right\}+\hat{W} \boldsymbol{I}=\left\{\left(J_{2} \hat{W}_{2}+\hat{W} / 2\right) \boldsymbol{I}+\hat{W}_{1} \hat{\boldsymbol{B}}+\hat{W}_{3} \hat{\boldsymbol{b}} \otimes \hat{\boldsymbol{b}}\right\}$,

where $\hat{W}\left(J_{1}, J_{2}, J_{3}\right)$ represents the two-dimensional version of the strain energy for growth and

$\hat{W}_{n}=\frac{\partial \hat{W}\left(J_{1}, J_{2}, J_{3}\right)}{\partial J_{n}} \quad(n=1,2,3)$.

Under the previously adopted assumption that mass-growth takes place in a prestress free manner $\left(\boldsymbol{T}_{0}=0\right),(8.8)$ enables the two-dimensional counterpart of the initial conditions (4.7) and (4.8) to yield

$\hat{W}(2,1,1)=\hat{W}_{0}, \quad \hat{W}_{3}(2,1,1)=0, \quad \hat{W}_{1}(2,1,1)+\hat{W}_{2}(2,1,1)+\hat{W}_{0} / 2=0$. 
When connected with $(8.4 \mathrm{~b})$ and $(8.7),(8.8)$ provides the in-plane components of the Cauchy stress as follows:

$$
\begin{aligned}
& \sigma_{r r}=2 J_{2} \hat{W}_{2}+\hat{W}+2\left(\hat{W}_{1}+\hat{W}_{3} A_{R}^{2}\right) \chi_{r}^{2}, \\
& \sigma_{\theta \theta}=2 J_{2} \hat{W}_{2}+\hat{W}+2\left(\hat{W}_{1}+\hat{W}_{3} A_{\Theta}^{2}\right) \chi_{\theta}^{2}+2 \gamma\left[\gamma\left(\hat{W}_{1}+\hat{W}_{3} A_{R}^{2}\right)+2 \chi_{\theta} \hat{W}_{3} A_{R} A_{\Theta}\right], \\
& \sigma_{r \theta}=2 \chi_{r}\left[\gamma\left(\hat{W}_{1}+\hat{W}_{3} A_{R^{2}}\right)+\chi_{\theta} \hat{W}_{3} A_{R} A_{\Theta}\right] .
\end{aligned}
$$

The prevailing axial symmetry considerations enable the radial and azimuthal equations of motion (2.12a, b) to simplify as follows:

$\sigma_{r r, r}=\left(\sigma_{\theta \theta}-\sigma_{r r}\right) / r, \quad\left(r^{2} \sigma_{r \theta}\right)_{, r}=0$,

while the axial equilibrium equation (2.12c) is satisfied identically. In a manner similar to that detailed in Sect. 5, integration of ( $8.12 \mathrm{~b}$ ) yields again (5.12a). Connection of the latter with the homogeneous version of the boundary condition yields then again $(5.14 \mathrm{a})$ which, by virtue of $(8.11 \mathrm{c})$, leads to

$R g_{, R}=\frac{\gamma}{\chi_{\theta}}=-\frac{\hat{W}_{3} A_{R} A_{\Theta}}{\hat{W}_{1}+\hat{W}_{3} A_{R}^{2}}$,

and, hence, to

$g(R ; t)=-\int_{A}^{R} \frac{\hat{W}_{3} A_{R} A_{\Theta} \mathrm{d} R}{R\left(\hat{W}_{1}+\hat{W}_{3} A_{R}^{2}\right)}$.

It is observed that (8.14) does satisfy the initial condition (2.3b) if use is made of a form of $\hat{W}$ that satisfies (8.10b). Moreover, (8.14) predicts that $g=0$ and, therefore, that there is absence of azimuthal shear deformation $(\gamma=0)$ if the fibres are straight radial $\left(A_{\Theta}=0\right)$ or concentric circles aligned with the azimuthal direction of the tube cross section $\left(A_{R}=0\right)$. However, the general case of spiral fibres $\left(A_{R} A_{\Theta} \neq 0\right)$ involves always generation of non-zero azimuthal shear deformation. These three particular cases are accordingly considered and discussed next separately.

\subsection{Radial fibres $\left(A_{\Theta}=0\right)$}

Because $\left(A_{\mathrm{R}}, A_{\Theta}\right)=(1,0)$ in this case, classified as $\left(i i_{1}\right)$ in Sect. 3, (8.14) yields $g=\gamma=0$, and the relevant restrictions (3.15) are satisfied identically. It is thus concluded that radial fibres do maintain their direction and shape, which is made alternatively obvious by observing that (8.6) returns

$\hat{\boldsymbol{b}}=\left\{\chi_{r}, 0\right\}^{\mathrm{T}}$.

Moreover, $\hat{\boldsymbol{B}}$ obtains a diagonal form, in which $\chi_{r}$ and $\chi_{\theta}$ are the principal growth stretches, and the invariants (8.7) simplify as follows:

$J_{1}=\chi_{r}^{2}+\chi_{\theta}^{2}, \quad J_{2}=\chi_{r}^{2} \chi_{\theta}^{2}, \quad J_{3}=\chi_{r}^{2}$.

By inserting (8.13) into (8.11), one obtains next the non-zero stress components as follows:

$$
\sigma_{r r}=2 J_{2} \hat{W}_{2}+\hat{W}+2\left(\hat{W}_{1}+\hat{W}_{3}\right) \chi_{r}^{2}, \quad \sigma_{\theta \theta}=2 J_{2} \hat{W}_{2}+\hat{W}+2 \hat{W}_{1} \chi_{\theta}^{2} .
$$


Upon inserting these stresses into the right-hand side of the radial equilibrium equation (8.12a), integration with respect to $r$ yields the radial normal stress in the following alternative form:

$\sigma_{r r}=q_{a}+2 \int_{a}^{r} \frac{1}{r}\left\{\hat{W}_{1} \chi_{\theta}^{2}-\left(\hat{W}_{1}+\hat{W}_{3}\right) \chi_{r}^{2}\right\} \mathrm{d} r$

where the boundary condition (2.13a) is also made use of. Hence, further combination of (8.18) and (8.12a) enables the hoop stress to also obtain an alternative form, which is as follows:

$\sigma_{\theta \theta}=q_{a}+2 \int_{a}^{r} \frac{1}{r}\left\{\hat{W}_{1} \chi_{\theta}^{2}-\left(\hat{W}_{1}+\hat{W}_{3}\right) \chi_{r}^{2}\right\} \mathrm{d} r+2\left\{\hat{W}_{1} \chi_{\theta}^{2}-\left(\hat{W}_{1}+\hat{W}_{3}\right) \chi_{r}^{2}\right\}$.

By connecting (8.18) with the homogeneous version of the remaining traction boundary condition, namely (2.13b) with $q_{b}=0$, it is finally found that

$q_{a}(t)=-2 \int_{a}^{b} \frac{1}{r}\left\{\hat{W}_{1} \chi_{\theta}^{2}-\left(\hat{W}_{1}+\hat{W}_{3}\right) \chi_{r}^{2}\right\} \mathrm{d} r$

The outer boundary of the tube can thus be kept free of external tractions, only if the inner boundary is supported by the externally applied pressure (8.20).

However, if the growing cross section is solid rather than hollow, then the growing long cylinder is indeed free of external tractions. Because $a=0$ in that case, $r(R ; t)$ and $\hat{W}$ are only required to be such that $(8.20)$ returns a finite value for $q_{a}(t)$. By virtue of (8.18) and (8.20) that value of $q_{a}(t)$ will then represent the finite value that the radial normal stress attains at the centre of the cross section.

The form of the unknown function $r(R ; t)$ may be determined by inserting (8.17) into the radial equilibrium equation (8.12a), and then solving the resulting differential equation, namely,

$r \frac{\partial R}{\partial r}\left[\hat{W} / 2+J_{2} \hat{W}_{2}+\left(\hat{W}_{1}+\hat{W}_{3}\right) \chi_{r}^{2}\right]_{, R}=\hat{W}_{1} \chi_{\theta}^{2}-\left(\hat{W}_{1}+\hat{W}_{3}\right) \chi_{r}^{2}$.

After suitable use of (8.16), it is observed with interest that forms of $\hat{W}$ that satisfy the condition

$$
\frac{\hat{W}_{3}}{\hat{W}_{1}}=\frac{J_{1}}{J_{3}}-2
$$

enable the radially reinforced long tube to grow in the completely stress-free manner $\left(\sigma_{r r}=\sigma_{\theta \theta}=\sigma_{r \theta}=0\right)$, which is also consistent with the adopted zero prestress consideration. It can be verified that (8.22) is consistent with the initial conditions (8.10).

The initial value problem (8.22) and (8.10) admits the relatively simple solution

$$
\hat{W}\left(J_{1}, J_{2}, J_{3}\right)=\left[J_{3}\left(J_{1}-J_{3}\right)-1\right] V\left(J_{2}\right)+\hat{W}_{0},
$$

where the otherwise arbitrary function $V\left(J_{2}\right)$ should be such that

$$
V(1)=-\hat{W}_{0} / 2
$$


One of the simplest possible choices of (8.23) is accordingly as follows:

$\hat{W}\left(J_{1}, J_{2}, J_{3}\right)=\left[J_{3}\left(J_{1}-J_{3}\right)-1\right]\left(J_{2}-1-\hat{W}_{0} / 2\right)$.

On the other hand, satisfaction of (8.22) enables (8.21) to simplify as follows:

$\left[\hat{W} / 2+J_{2} \hat{W}_{2}+\hat{W}_{1} \chi_{\theta}^{2}\right]_{, R}=0$.

This is satisfied identically in cases of radially homogeneous mass-growth, namely,

$r(R ; t)=R \varphi(t), \varphi\left(t_{0}\right)=1$,

where the strain invariants (8.16) depend only on time and, therefore, $\hat{W}_{, R}=0$.

\subsection{Circumferential fibres $\left(A_{R}=0\right)$}

Because $\left(A_{R}, A_{\Theta}\right)=(0,1)$ in this particular case, (8.14) yields again $g=\gamma=0$. The invariants $(8.7 \mathrm{a}, \mathrm{b})$ attain again the simplified form $(8.16 \mathrm{a}, \mathrm{b})$, respectively, but the third becomes $J_{3}=\chi_{\theta}^{2}$. Hence, (8.6) yields

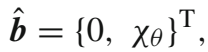

thus showing that, indeed, concentric circular fibres maintain their shape during axisymmetric mass-growth.

In this case, the basic governing differential equation (8.12a) reduces to

$r \frac{\partial R}{\partial r}\left[\hat{W} / 2+J_{2} \hat{W}_{2}+\hat{W}_{1} \chi_{r}^{2}\right]_{, \boldsymbol{R}}=\left(\hat{W}_{1}+\hat{W}_{3}\right) \chi_{\theta}^{2}-\hat{W}_{1} \chi_{r}^{2}$.

Moreover, the process detailed above for the derivation of (8.19) and (8.20) yields now the non-zero in-plane stress components as follows:

$$
\begin{aligned}
& \sigma_{r r}=q_{a}+2 \int_{a}^{r} \frac{1}{r}\left\{\left(\hat{W}_{1}+\hat{W}_{3}\right) \chi_{\theta}^{2}-\hat{W}_{1} \chi_{r}^{2}\right\} \mathrm{d} r, \\
& \sigma_{\theta \theta}=q_{a}+2 \int_{a}^{r} \frac{1}{r}\left\{\left(\hat{W}_{1}+\hat{W}_{3}\right) \chi_{\theta}^{2}-\hat{W}_{1} \chi_{r}^{2}\right\} \mathrm{d} r+2\left\{\left(\hat{W}_{1}+\hat{W}_{3}\right) \chi_{\theta}^{2}-\hat{W}_{1} \chi_{r}^{2}\right\},
\end{aligned}
$$

where application on the inner tube boundary of the external pressure

$q_{a}(t)=-2 \int_{a}^{b} \frac{1}{r}\left\{\left(\hat{W}_{1}+\hat{W}_{3}\right) \chi_{\theta}^{2}-\hat{W}_{1} \chi_{r}^{2}\right\} \mathrm{d} r$,

can keep again the outer tube boundary free of external tractions.

It is then observed that forms of $\hat{W}$ that satisfy (8.22) yield now

$$
\left(\hat{W}_{1}+\hat{W}_{3}\right) \chi_{\theta}^{2}-\hat{W}_{1} \chi_{r}^{2}=0
$$


and, hence, enable again axisymmetric cross-sectional mass-growth to take place in a stress-free manner. Such forms of $\hat{W}$ enable further (8.29) to obtain the simplified form

$\left[J_{2} \hat{W}_{2}+\hat{W} / 2+\hat{W}_{1} \chi_{r}^{2}\right]_{, \boldsymbol{R}}=0$

which is again satisfied identically in the particular case of radially homogeneous mass-growth (8.27).

It follows that if (8.22) and (8.27) hold simultaneously under a mass-growth rate that satisfies (8.5), then the cross section of a radially or a circumferentially reinforced infinitely long tube subjected to homogeneous traction boundary conditions $\left(q_{a}=q_{b}=\tau_{b \theta}=0\right)$ maintains purely radial, inflation-type mass-growth $(g=\gamma=0)$ in a completely stress-free manner.

\subsection{Cross-sectional spiral fibres $\left(A_{R} A_{\Theta} \neq 0\right)$}

As is concluded in Sect. 3, cross-sectional spiral fibres maintain their shape and direction during the tube mass growth only if (3.17) are satisfied. While the first of these conditions is satisfied by the mass-growth pattern (8.2), the second may be converted into the following:

$r_{, R}-\left(g_{, R} A_{R} / A_{\Theta}+1 / R\right) r=0$.

When the fibre direction $\boldsymbol{A}$ is independent of the radial coordinate parameter and, therefore, constant, the differential equation (8.34) admits the general solution

$r(R ; t)=R \varphi(t) \exp \left(g_{, R} A_{R} / A_{\Theta}\right)=R \varphi(t) \exp \left(-A_{R}^{2} \int_{A}^{R} \frac{\mathrm{d} R}{\left(A_{R}^{2}+\hat{W}_{1} / \hat{W}_{3}\right) R}\right)$,

where (8.14) is also made use of. Here, $\varphi(t)$ represents an arbitrary non-dimensional function of time which, by virtue of (8.10b), is still required to conform with the initial condition $(8.27 \mathrm{~b})$.

The radially non-linear form of (8.35) represents a non-homogeneous mass-growth deformation pattern, which yields

$\chi_{\theta}=\varphi(t) \exp \left(-A_{R}^{2} \int_{A}^{R} \frac{\mathrm{d} R}{\left(A_{R}^{2}+\hat{W}_{1} / \hat{W}_{3}\right) R}\right), \quad \chi_{r}=\chi_{\theta} \frac{\hat{W}_{1}}{\hat{W}_{1}+\hat{W}_{3} A_{R^{2}}}, \quad \gamma=-\chi_{\theta} \frac{\hat{W}_{3} A_{R} A_{\Theta}}{\hat{W}_{1}+\hat{W}_{3} A_{R}^{2}}$.

Relevant forms of the deformation invariants are obtained by inserting these expression into (8.7). Moreover, after use of (8.11) and lengthy algebraic manipulations, the radial equation of motion (8.12a) attains the relatively simple form

$R\left\lfloor J_{2} \hat{W}_{2}+\sqrt{J_{2}} \hat{W}_{1}+\hat{W} / 2\right\rfloor_{, R}=\hat{W}_{3}$.

It is accordingly seen that (8.14), (8.35) and (8.37) form a set of three simultaneous equations for the three principal unknown functions $r(R ; t), g(R ; t)$ and $\hat{W}\left(J_{1}, J_{2}, J_{3}\right)$. Alternatively, use of (8.14) in connection with (8.36) may convert (8.37) into a single equation for $\hat{W}$. Potential solution of that highly non-linear integro-differential equation may then provide $r(R ; t)$ and $g(R ; t)$ through subsequent substitution into (8.35) and (8.14), respectively. However, such a solution will not be pursued here any further. It should be noted that the incompressible mass-growth process 
of present interest may become possible only if associated with a rate of mass-growth which is obtained by inserting (8.35) into the right- hand side of (8.5).

\section{Conclusions}

Several types of tube-like fibre-reinforced tissue have the ability to grow in a manner that preserves not only their shape, but also the shape and direction of their embedded fibres. Motivated by this observation, this investigation considered the most general axisymmetric mass-growth pattern of a finite tube reinforced by a single family of fibres, and investigated the influence that preservation of the fibre direction exerts on relevant mathematical modelling, as well as on the growth mechanism and the physical behaviour of the tube. Accordingly, relevant sets of necessary conditions that enable axisymmetric mass-growth patterns of a tube to take place were developed not only for fibres that preserve some general direction, but also for all six particular cases in which fibre direction remains normal to either one or two of the associated cylindrical polar coordinates.

Those conditions exert direct influence only on the kinematic characteristics of the tube growth pattern. They are mathematically independent of the tube material features and behaviour, and are thus required to hold in addition to and, therefore, simultaneously with the standard stress equilibrium equations. By enhancing coupling between tube kinematics and constitutive characteristics, they thus reflect the hidden ability of the growing tube to guide its material behaviour in a manner that enables the embedded growing fibres to preserve direction and shape. Those conditions can thus be exploited, and employed in a manner that provides valuable information regarding the constitutional behaviour of a fibre-reinforced tube undergoing axisymmetric mass-growth.

It is accordingly seen with examples that, in cases that validity of (4.3) and (4.5) justify the consideration and the use of the concept of mass-growth hyperelasticity, the additional information provided by the aforementioned conditions enables identification of specific classes of the strain energy density for growth that are suitable and, hence, admissible in different types of axisymmetric tissue mass-growth. In this context, the example applications considered in Sects. 5-8 refer to different tube mass-growth patterns that take place in an incompressible manner and, hence, enable the continuity equation with growing mass to attain the simplified form (4.3).

This form of the continuity equation exerts direct influence on the dilatational features of the mass-growth of interest and leads naturally to (5.6), which relates the fibre growth stretch with the rate of growth of the tube material. The predominantly dilatational nature of the observed mass-growth pattern identifies thus the fibre growth stretch as the principal proportionality parameter that dictates not only the purely dilatational mass-growth pattern discussed in Sect. 5, but also the combined dilatational and shear types of growth detailed afterwards in Sects. 6 and 7. It is emphasised that, due to their different kinematic characteristics, each of the three-dimensional mass-growth patterns studied in Sects. 5-7 is associated with some different class or classes of admissible strain energy density for growth.

However, the example growth patterns studied in Sects. 5 and 6 are both triggered by the same class of mass-growth rates and are applicable regardless of the preserved fibre shape and direction. They lead to identical predictions of fibre growth stretch and, hence, they both preserve during growth the initial ratio between the radial and axial tube dimensions. Mass-growth patterns sought and found in Sect. 6 are accordingly regarded as alternative or additional to their purely dilatational counterparts developed previously in Sect. 5 in the sense that, due to availability and use of a different class of admissible strain energy densities for growth, they superpose on mass-growth dilatation appropriate amounts of torsional deformation.

In contrast, the example application discussed in Sect. 7 focuses attention on a specific shape of fibres. This is the shape of fibres that grow with and within the tube tissue in a helical manner and, as is detailed in the Introduction, met often in nature. This application enables thus the identification of strain energy densities for growth that, as is also observed in nature, allow the tube to grow in a manner that does not preserve the ratio between its radial and axial dimensions.

Several of the outlined observations still hold in the last application considered in Sect. 8 which, however, considered and studied the cross-sectional, plane strain mass-growth of a transversely isotropic tube of infinite 
extent. An interesting case of stress-free cross-sectional mass-growth has thus been identified when the infinitely long tube of interest is reinforced by a radial (Sect. 8.1) or circumferential family of fibres (Sect. 8.2). Such stressfree mass-growth has the form of a homogeneous axisymmetric deformation and is possible only if the strain energy for growth belongs to a class of functions which, like (8.23) or (8.25), satisfy the differential equation (8.22). Moreover, it fits in an exact mathematical manner the quasi-static equilibrium framework considered, as long as that deformation is radially uniform and linearly proportional to time.

Most interestingly, this kind of homogeneous, stress-free mass-growth is possible in the case of a finite tube regardless of the fibre shape and direction, provided that the strain energy for growth satisfies simultaneously the differential equations (5.15) and (5.23). This is however not possible in cases that the infinitely long tube of interest is reinforced by a cross-sectional family of spiral fibres. Section 8.3 makes thus available a non-homogeneous mass-growth pattern that enables the present analysis to also consider and account for cross-sectional tube massgrowth that preserves the direction of spiral fibres, namely, plane cross-sectional fibres which are neither radial nor circumferential.

There is a large number of additional mass-growth applications that may be considered and studied in a manner similar to that detailed in Sects. 5-8. These may be formed by considering, for instance, several different combinations of non-zero components in the deformation tensors (2.4). Consideration and study of any of those potential axisymmetric mass-growth patterns in association with some particular fibre direction met in nature, like the helical fibre direction considered in Sect. 7, will also be of interest. Moreover, cases of non-axisymmetric mass-growth of tube-like fibre-reinforced tissue may possibly be met in nature, and could be considered and modelled in a similar manner.

Along with the type of the incompressible hyperelastic mass-growth introduced in Sect. 4, there should exist in nature mass-growth processes which are compressible. Rather than (4.3), such types of growth make use of the full form of the continuity equation with growing mass (2.10) and may thus be able to influence directly not only the dilatational, but also the shear deformation features of axisymmetric tube mass-growth. The present hyperelasticity mass-growth model can then be found helpful in directing relevant compressible mass-growth developments [22,32], where the influence of the divergence of the velocity vector is still accounted for in the corresponding constitutive equation (4.1). Similarly, the presented analysis may assist future developments in which plastic-like mass-growth and/or influence of some non-zero prestress state are further expected to be accounted for $[22,29]$.

A final comment should refer to relevant tube growth cases that involve more than one family of growing fibres. Most likely, consideration of such cases in association with the postulates and ideas described in the present investigation will increase the number of the kinematic restrictions outlined in Sect. 3.2. As a result, the aforementioned strain energy density classes should be expected to become narrower and, potentially, lead thus to simpler forms of admissible strain energy densities for growth. Such a potential result should not be considered as very restrictive for the mechanical behaviour of a growing tube-like tissue because, as is justified in Sect. 4, the strain energy density for growth and the strain energy density for mechanically caused deformations of the same tissue should, in general, not be the same.

Open Access This article is distributed under the terms of the Creative Commons Attribution 4.0 International License (http:// creativecommons.org/licenses/by/4.0/), which permits unrestricted use, distribution, and reproduction in any medium, provided you give appropriate credit to the original author(s) and the source, provide a link to the Creative Commons license, and indicate if changes were made.

\section{Appendix A: Preservation of fibre direction if radial growth is independent of the axial coordinate}

Consider radial growth which does not depend on the axial tube parameter, $Z$, namely a particular case in which (2.2) simplifies as follows

$r=r(R ; t), \quad \theta=\Theta+g(R, Z ; t), \quad z=z(R, Z ; t)$. 
Because $\gamma_{r z}=0$ in this case, the general constraint conditions (3.5) simplify and become

$A_{R}\left[\gamma_{\theta r} A_{R}+\left(\chi_{\theta}-\chi_{r}\right) A_{\Theta}+\gamma_{\theta z} A_{Z}\right]=0$,

$A_{R}\left[\gamma_{z r} A_{R}+\left(\chi_{z}-\chi_{r}\right) A_{Z}\right]=0$

$\gamma_{\theta z} A_{Z}^{2}+\left(\chi_{\theta}-\chi_{z}\right) A_{\Theta} A_{Z}+\left(\gamma_{\theta r} A_{Z}-\gamma_{z r} A_{\Theta}\right) A_{R}=0$,

while their explicit equivalent form (3.6) reduces to

$r g_{, R}+\left(r / R-r_{, R}\right)\left(A_{\Theta} / A_{R}\right)+r g_{, Z}\left(A_{Z} / A_{R}\right)=0, \quad z_{, R}+\left(z, Z-r_{, R}\right)\left(A_{Z} / A_{R}\right)=0$.

This simplification allows further progress to be made even in the general case in which validity of (3.3) suggests that none of the cylindrical polar coordinates is perpendicular to the fibre direction.

Accordingly, elimination of $r_{, R}$ in (A.3) yields the following differential relationship between $g(R, Z ; t)$ and $z=z(R, Z ; t)$ :

$z, Z+z, R A_{R} / A_{Z}=1 / R+g_{, Z} A_{Z} / A_{\Theta}+g_{, R} A_{R} / A_{\Theta}$.

Moreover, appropriate rearrangement of (A.3b) provides a relevant relationship between $r(R ; t)$ and $g(R, Z ; t)$, namely,

$r_{, R}=z, Z+z, R A_{R} / A_{Z}$.

It can readily be verified that these results are also consistent with all sets of constraint conditions detailed in Sect. 3.2 for fibre directions that violate (3.3).

\section{Appendix B: Formulation of the constitutive equation (4.1)}

Formulation of constitutive equation (4.1) begins by proposing the usual energy balance equation

$\frac{\mathrm{d}}{\mathrm{dt}} \int_{V} W \mathrm{~d} V=\int_{S} \boldsymbol{t} \cdot \boldsymbol{v} \mathrm{d} S$

where the Cartesian components of the traction vector $\boldsymbol{t}$ are given through the Cauchy's formula

$t_{\mathrm{i}}=\sigma_{j i} n_{j}$,

where $\boldsymbol{n}$ stands for the outward unit normal on the surface $S$ that encloses an arbitrary volume $V$ in the current configuration of the continuum of interest. Application of the divergence theorem and the Reynolds' transport theorem for a continuum with growing mass [33] yields

$\frac{1}{\rho_{0}}\left(\rho \dot{W}+r_{g} W\right)=\sigma_{j i} v_{i, j}+v_{i} \sigma_{j i, j}=\sigma_{j i} d_{i j}$,

where the Cartesian form of the quasi-static equilibrium equations (2.11)is made use of. Hence, (B.3) reduces further to the following:

$\frac{\rho}{\rho_{0}} \dot{W}=\left(\sigma_{j i}-\frac{r_{g}}{v_{k, k}} W \delta_{i j}\right) d_{i j}$. 
Considering on the other hand that, in a usual manner, $W=W(\boldsymbol{C})$, one obtains

$$
\begin{aligned}
\dot{W} & =\frac{\partial W}{\partial C_{R S}} \frac{d C_{R S}}{d t}=\frac{\partial W}{\partial C_{R S}} \frac{d}{d t}\left(\frac{\partial x_{i}}{\partial X_{R}} \frac{\partial x_{i}}{\partial X_{S}}\right)=\frac{\partial W}{\partial C_{R S}}\left(\frac{\partial v_{i}}{\partial X_{R}} \frac{\partial x_{i}}{\partial X_{S}}+\frac{\partial x_{i}}{\partial X_{R}} \frac{\partial v_{i}}{\partial X_{S}}\right) \\
& =\left(\frac{\partial W}{\partial C_{R S}}+\frac{\partial W}{\partial C_{S R}}\right) \frac{\partial x_{i}}{\partial X_{R}} \frac{\partial v_{i}}{\partial X_{S}}=\left(\frac{\partial W}{\partial C_{R S}}+\frac{\partial W}{\partial C_{S R}}\right) \frac{\partial x_{i}}{\partial X_{R}} \frac{\partial x_{j}}{\partial X_{S}} \frac{\partial v_{i}}{\partial x_{j}} \\
& =F_{i R} F_{j S}\left(\frac{\partial W}{\partial C_{R S}}+\frac{\partial W}{\partial C_{S R}}\right) \frac{\partial v_{i}}{\partial x_{j}}=F_{i R} F_{j S}\left(\frac{\partial W}{\partial C_{R S}}+\frac{\partial W}{\partial C_{S R}}\right) d_{i j} .
\end{aligned}
$$

Then, a comparison with (B.4) yields the constitutive Equation (4.1).

\section{Appendix C: Adherence to the quasi-static growth concept}

Mass-growth processes may be considered quasi-static if they involve negligibly small acceleration terms and, hence, can support the claim that time variation of the velocity components (2.7) is also negligible. In dealing with the purely dilatational mass-growth problem considered in Sect. 5, the choice

$\lambda(t)=t / t_{0}$,

is thus considered optimal because it yields the velocity components in the following time-independent form:

$v_{r}=R / t_{0}, \quad v_{\theta}=0, \quad v_{z}=Z / t_{0}$.

However, by virtue of (5.7) or, equivalently, (5.6), (C.1) can be associated with the linearly proportional dilatational growth (5.5) only if the rate of growth has the form

$r_{g}=3 \rho_{0} / t$

which is inversely proportional to time. Moreover, (6.6) as well as (7.1) and (7.5) implies that (C.1), (C.2a) and (C.2c) may still be associated with the mass-growth processes discussed in Sects. 6 and 7 where, however, the appearance and influence of relevant shear strain is further taken into consideration.

In this context, the non-zero azimuthal placement component (6.9) gives rise to the azimuthal velocity component (6.12) in Sect. 6 which, when connected with the boundary condition (2.14a), leads to the relationship

$\phi(t)=\frac{\alpha(Z ; t)}{A A_{Z}-Z A_{R}}$.

Hence, when the fibre growth stretch is given according to (C.1), then (6.12) simplifies into the following:

$v_{\theta}=\dot{\alpha} \frac{t R\left(R A_{Z}-Z A_{R}\right)}{t_{0}\left(A A_{Z}-Z A_{R}\right)}$

This form of $v_{\theta}$ can be independent of time and, hence, strictly compatible with quasi-static mass-growth considerations, only if $\alpha$ is a logarithmic function of time. It follows that the choice

$\alpha(Z ; t)=\left(A A_{Z}-Z A_{R}\right) \ln \left(t / t_{0}\right)$, 
provides a unique definition of the otherwise arbitrary function $\phi(t)$ employed in either Sects. 6 and 7, namely,

$\phi(t)=\ln \left(t / t_{0}\right)$.

It is noted that the choices (C.1) and (C.6) enable satisfaction of both initial conditions (6.6c) and (6.9b), and are thus consistent with the example application considered in Sect. 6. These choices fit also well the application considered in Sect. 7 where, however, they do not satisfy the concept of quasi-static growth in an exact manner.

\section{Appendix D: On admissible forms of $W$ for combined dilatational and torsional mass-growth}

Forms of $W$ which are admissible for the mass-growth application discussed in Sect. 6 should satisfy the PDE (6.16). This condition may be converted into a second-order linear PDE for $W$, as soon as the denoted partial differentiation with respect to $R$ is carried out with the help of the following intermediate result:

$$
\begin{aligned}
\frac{\partial W_{n}}{\partial R} & =\sum_{m=1}^{5} W_{n m} \frac{\partial I_{m}}{\partial R}=\left(W_{n 1}+\lambda^{2} W_{n 2}+\lambda^{2} A_{\Theta}^{2} W_{n 5}\right) \frac{\partial I_{1}}{\partial R} \\
& =2\left(W_{n 1}+\lambda^{2} W_{n 2}+\lambda^{2} A_{\Theta}^{2} W_{n 5}\right) \lambda^{2} \phi^{2} R\left(1-A_{\Theta}^{2}\right),
\end{aligned}
$$

where the following notation is employed:

$W_{n m}=\frac{\partial W_{n}}{\partial I_{m}}=\frac{\partial^{2} W}{\partial I_{n} \partial I_{m}} \quad(n, m=1,2, \ldots, 5)$,

and the relationships

$$
\frac{\partial I_{1}}{\partial R}=\lambda^{-2} \frac{\partial I_{2}}{\partial R}=\left(\lambda A_{\Theta}\right)^{-2} \frac{\partial I_{5}}{\partial R}=2 \lambda^{2} \phi^{2} R\left(1-A_{\Theta}^{2}\right), \quad \frac{\partial I_{3}}{\partial R}=\frac{\partial I_{4}}{\partial R}=0
$$

are also made use of. Nevertheless, the implied PDE for $W$ should hold simultaneously with the pair of conditions (6.14) and may thus be simplified accordingly.

In a relatively simple illustrating example, one may look, for instance, for admissible forms of $W$ which are independent of the strain invariant $I_{5}$ and, therefore,

$W_{5}=W_{n 5}=0$.

In that case, (6.14) yield

$$
\left(\begin{array}{l}
W_{4} \\
W_{1}
\end{array}\right)=\left(\begin{array}{c}
\alpha \\
\beta
\end{array}\right) W_{2}
$$

where the appearing proportionality factors are

$\alpha=-\lambda^{2} \phi^{2} R^{2}, \beta=\lambda^{2}\left(\phi R \frac{A_{R} A_{\Theta}}{A_{Z}}-1\right)$.

As a result, (6.16) may initially simplify into the following:

$$
\begin{aligned}
& R\left\{\lambda^{6} W_{3, R}+\frac{1}{2} W_{, R}+\lambda^{4}\left(1+\phi R \frac{A_{R} A_{\Theta}}{A_{Z}}\right) W_{2, R}+\lambda^{4} \phi \frac{A_{R} A_{\Theta}}{A_{Z}} W_{2}\right\} \\
& =\lambda^{2} \phi^{2} R^{2}\left[\phi R \frac{A_{R}}{A_{Z}} A_{\Theta}\left(1-A_{\Theta^{2}}\right)-A_{\Theta}^{2}\right] W_{2} .
\end{aligned}
$$


With use of (D.1), (D.2), (D.4b), (D.5) and the intermediate result

$\frac{\partial \beta}{\partial I_{2}}=\frac{\left[2 \phi^{2} R^{2}\left(1-A_{\Theta}^{2}\right)+3\right]-\phi R\left(1-A_{\Theta}^{2}\right) A_{Z}}{2 \lambda^{2} \phi R\left(1-A_{\Theta}^{2}\right) A_{Z}\left[\phi^{2} R^{2}\left(1-A_{\Theta}^{2}\right)+3\right]}$,

(D.7) may next be brought into the following form of a second-order linear PDE

$f_{1} W_{22}+f_{2} W_{23}+f_{3} W_{2}=0$

where the appearing variable coefficients are as follows:

$$
\begin{aligned}
f_{1}= & \lambda^{6} \phi R \frac{A_{R}}{A_{Z}} A_{\Theta}\left(\phi R \frac{A_{R} A_{\Theta}}{A_{Z}}+1\right), \quad f_{2}=\lambda^{8} \phi R \frac{A_{R} A_{\Theta}}{A_{Z}}, \\
f_{3}= & \lambda^{2}\left\{\phi R \frac{A_{R} A_{\Theta}}{3 A_{Z}}-\frac{5}{6}+\frac{A_{\Theta}}{2\left(1-A_{\Theta}^{2}\right)}\left(\frac{A_{R}}{\phi R A_{Z}}+A_{\Theta}\right)\right. \\
& \left.+\left(1+\phi R \frac{A_{R} A_{\Theta}}{A_{Z}}\right) \frac{\left[2 \phi^{2} R^{2}\left(1-A_{\Theta}^{2}\right)+3\right] A_{R} A_{\Theta}-\phi R\left(1-A_{\Theta}^{2}\right) A_{Z}}{2 \phi R\left(1-A_{\Theta}^{2}\right) A_{Z}\left[\phi^{2} R^{2}\left(1-A_{\Theta}^{2}\right)+3\right]}\right\} .
\end{aligned}
$$

By virtue of (6.11), it is next seen that

$$
\lambda^{2}=I_{4}=I_{3}^{1 / 3}, \quad \phi^{2} R^{2}=\frac{I_{1} I_{4}^{-1}-3}{1-A_{\Theta}^{2}}=\frac{I_{2} I_{4}^{-2}-3}{1-A_{\Theta}^{2}}=\frac{I_{5} I_{4}^{-2}-1}{\left(1-A_{\Theta}^{2}\right) A_{\Theta}^{2}}=\frac{I_{1} I_{3}^{-1 / 3}-3}{1-A_{\Theta}^{2}}=\frac{I_{2} I_{3}^{-2 / 3}-3}{1-A_{\Theta}^{2}}=\frac{I_{5} I_{3}^{-2 / 3}-1}{\left(1-A_{\Theta}^{2}\right) A_{\Theta}^{2}}
$$

and, hence, the variable factors defined in (D.6) and (D.10) can be converted into functions of the strain invariants in several different ways.

By employing, for instance, the option

$\lambda^{2}=I_{4}, \quad \phi^{2} R^{2}=\frac{I_{1} I_{4}^{-1}-3}{1-A_{\Theta}^{2}}$,

(D.6) and (D.10) obtain the following, more general form:

$$
\begin{aligned}
& \alpha\left(I_{1}, I_{4}\right)=\frac{3 I_{4}-I_{1}}{1-A_{\Theta}^{2}}, \\
& \beta\left(I_{1}, I_{4}\right)=\frac{A_{R} A_{\Theta}\left(I_{1} I_{4}-3 I_{4}^{2}\right)^{1 / 2}}{A_{Z}\left(1-A_{\Theta}^{2}\right)^{1 / 2}}-I_{4}, \\
& f_{1}\left(I_{1}, I_{4}\right)=I_{4}^{3} \sqrt{\frac{I_{1} I_{4}^{-1}-3}{1-A_{\Theta}^{2}}} \frac{A_{R} A_{\Theta}}{A_{Z}}\left(\sqrt{\frac{I_{1} I_{4}^{-1}-3}{1-A_{\Theta}^{2}}} \frac{A_{R} A_{\Theta}}{A_{Z}}+1\right) \text {, } \\
& f_{2}\left(I_{1}, I_{4}\right)=I_{4}^{4} \sqrt{\frac{I_{1} I_{4}^{-1}-3}{1-A_{\Theta}^{2}}} \frac{A_{R} A_{\Theta}}{A_{Z}},
\end{aligned}
$$

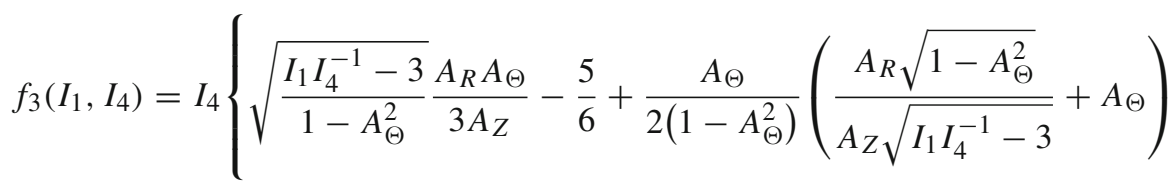

$$
\begin{aligned}
& \left.+I_{4}\left(1+\sqrt{\frac{I_{1} I_{4}^{-1}-3}{1-A_{\Theta}^{2}}} \frac{A_{R} A_{\Theta}}{A_{Z}}\right) \frac{\left(2 I_{1} I_{4}^{-1}-3\right) A_{R} A_{\Theta}-A_{Z} \sqrt{\left(I_{1} I_{4}^{-1}-3\right)\left(1-A_{\Theta}^{2}\right)}}{2 A_{Z} I_{1} \sqrt{\left(I_{1} I_{4}^{-1}-3\right)\left(1-A_{\Theta}^{2}\right)}}\right\}
\end{aligned}
$$

and, hence, (D.9) simplifies into the first-order linear PDE

$f_{1}\left(I_{1}, I_{4}\right) \hat{W}_{2}+f_{2}\left(I_{1}, I_{4}\right) \hat{W}_{3}+f_{3}\left(I_{1}, I_{4}\right) \hat{W}=0$, 
where

$\hat{W}\left(I_{1}, I_{4}, I_{3}, I_{4}\right)=W_{2}$

becomes the principal unknown function.

A search for a possible solution of the form

$\hat{W}\left(I_{1}, I_{2}, I_{3}, I_{4}\right)=\tilde{W}\left(I_{2}, I_{3}\right) \exp \left[\tilde{f}\left(I_{1}, I_{4}\right)\right]$,

where $\tilde{f}\left(I_{1}, I_{4}\right)$ is an arbitrary function of its arguments, then converts (D.14) into the following first-order PDE for $\tilde{W}$ :

$\frac{f_{1}\left(I_{1}, I_{4}\right)}{f_{3}\left(I_{1}, I_{4}\right)} \tilde{W}_{2}+\frac{f_{2}\left(I_{1}, I_{4}\right)}{f_{3}\left(I_{1}, I_{4}\right)} \tilde{W}_{3}+\tilde{W}=0$.

It may then readily be verified that this admits the separable solution

$\tilde{W}\left(I_{2}, I_{3}\right)=\tilde{c}_{1} \exp \left[\tilde{c}_{2} \frac{f_{3}\left(I_{1}, I_{4}\right)}{f_{1}\left(I_{1}, I_{4}\right)} I_{2}-\left(1+\tilde{c}_{2}\right) \frac{f_{3}\left(I_{1}, I_{4}\right)}{f_{2}\left(I_{1}, I_{4}\right)} I_{3}\right]$

where $\tilde{c}_{1}$ and $\tilde{c}_{2}$ are arbitrary constants. With the function $\hat{W}$ becoming thus available, a direct integration of (D.15) with respect to $I_{2}$ yields a relevant class of admissible energy densities in the form (6.17).

\section{Appendix E: On admissible forms of $W$ associated with growth of helical fibres}

By virtue of (7.12), (7.11c) simplifies into the following:

$R\left\{\lambda^{4} W_{3}+W / 2+\lambda^{2} W_{2}\right\}_{, R}=\left(4 \lambda^{2} \phi^{2} R^{2} W_{2} A_{Z}^{4}+W_{4} A_{\Theta}^{4}\right) / A_{\Theta}^{2}$.

This condition may be converted into the form of a linear PDE for $W$ after the partial differentiation with respect to $R$ is carried out with the help of the relationships

$\frac{\partial W}{\partial R}=\sum_{m=1}^{5} W_{m} \frac{\partial I_{m}}{\partial R}, \quad \frac{\partial W_{n}}{\partial R}=\sum_{m=1}^{5} W_{n m} \frac{\partial I_{m}}{\partial R} \quad(n=2,3)$.

However, (7.12b) implies that $W$ should be independent of $I_{5}$ and, therefore,

$W_{5}=W_{n 5}=0 \quad(n=2,3)$.

Moreover, it is

$\frac{\partial I_{2}}{\partial R}=\lambda^{2} \frac{\partial I_{1}}{\partial R}=\lambda^{4} 2 \phi^{2} R A_{Z}^{2}\left(1+4 A_{Z}^{2} / A_{\Theta}^{2}\right), \quad \frac{\partial I_{3}}{\partial R}=\frac{\partial I_{4}}{\partial R}=0$,

while validity of (7.12a) reveals further that

$\frac{\partial W}{\partial R}=\left(W_{1}+\lambda^{2} W_{2}\right) \frac{\partial I_{1}}{\partial R}=0, \quad \frac{\partial W_{n}}{\partial R}=\left(W_{n 1}+\lambda^{2} W_{n 2}\right) \frac{\partial I_{1}}{\partial R}=\frac{\partial I_{1}}{\partial R} \frac{\partial\left(W_{1}+\lambda^{2} W_{2}\right)}{\partial I_{n}}=0$. 
The left-hand side of (E.1) is thus shown equal to zero and, hence, its equivalent equation (7.11c) obtains the simplified form (7.13).

\section{References}

1. Barnett JR, Bonham VA (2004) Cellulose microfibril angle in the cell wall of wood fibres. Biol Rev 79:461-472

2. Reisinger AG, Pahr DH, Zysset PK (2011) Elastic anisotropy of bone lamellae as a function of fibril orientation pattern. Biomech Model Mechanobiol 10:67-77

3. Fournier M, Dlouhá J, Jaouen G, Almeras T (2013) Integrative biomechanics of tree ecology: beyond wood density and strength. J Exp Bot 64:4793-4815

4. Cyron CJ, Humphrey JD (2015) Preferred fiber orientations in healthy arteries and veins understood from netting analysis. Math Mech Solids 20:680-696

5. Holzapfel GA, Niestrawska JA, Ogden RW, Reinisch AJ, Schriefl AJ (2015) Modelling non-symmetric collagen fibre dispersion in arterial walls. J R Soc Interface 12:20150188

6. Qi N, Gao H, Ogden RW, Hill NA, Holzapfel GA, Han H-C, Luo X (2015) Investigation of the optimal collagen fibre orientation in human iliac arteries. J Mech Behav Biomed Mater 52:108-119

7. Schriefl AJ, Zeindlinger G, Pierce DM, Regitnig P, Holzapfel GA (2012) Determination of the layer-specific distributed collagen fibre orientations in human thoracic and abdominal aortas and common iliac arteries. J R Soc Interface 9:1275-1286

8. De Eguileor M, Valvassori R, Lanzavecchia G, Grimaldi A (1996) Morphogenesis of helical fibres in haplotaxids. Hydrobiologia 334:207-217

9. De Eguileor M, Grimaldi A, Lanzavecchia G, Tettamanti G, Valvassori R (1998) Dimensional and numerical growth of helical fibers in leeches: an unusual pattern. J Exp Zool A 281:171-187

10. Gutfreund Y, Flash T, Yarom Y, Fiorito G, Segev I, Hochner B (1996) Organization of octopus arm movements: a model system for studying the control of flexible arms. J Neurosci 16:7297-7307

11. Kier WM, Stella MP (2007) The arrangement and function of octopus arm musculature and connective tissue. J Morphol 268:831843

12. Fung YC, Fronek K, Patitucci P (1979) Pseudoelasticity of arteries and the choice of its mathematical expression. Am J Physiol 237:H620-631

13. Holzapfel GA, Gasser TC, Ogden RW (2000) A new constitutive framework for arterial wall mechanics and a comparative study of material models. J Elast 6:1-48

14. Humphrey JD (2003) Continuum biomechanics of soft biological tissues. Proc R Soc Lond A 453:3-46

15. Holzapfel GA, Ogden RW (eds) (2006) Mechanics of biological tissue. Springer, Berlin

16. Haughton DM, Merodio J (2009) The elasticity of arterial tissue affected by Marfan's syndrome. Mech Res Commun 36:659-668

17. Fu YB, Rogerson GA, Zhang YT (2012) Initiation of aneurysms as a mechanical bifurcation phenomenon. Int J Non Linear Mech 47:179-184

18. Jones GW, Chapman SJ (2012) Modeling in biological materials. SIAM Rev 54:52-118

19. Cowin SC (2004) Tissue growth and remodeling. Annu Rev Biomed Eng 6:77-107

20. Lubarda VA, Hoger A (2004) On the mechanics of solids with growing mass. Int J Solids Struct 39:4627-4664

21. Guillou A, Ogden RW (2006) Growth in soft biological tissue and residual stress development. In: Holzapfel GA, Ogden RW (eds) Mechanics of biological tissue. Springer, Berlin, pp 47-62

22. Soldatos KP (2013) Modelling framework for mass-growth. Mech Res Commun 50:50-57

23. Jiang X, Ogden RW (1998) On azimuthal shear of a circular cylindrical tube of compressible elastic material. Q J Mech Appl Math $51: 143-158$

24. Kassianidis F, Ogden RW, Merodio J, Pence TJ (2008) Azimuthal shear of a transversely isotropic elastic solid. Math Mech Solids 13:690-724

25. Soldatos KP (2010) Second-gradient plane deformations of ideal fibre-reinforced materials: implications of hyper-elasticity theory. J Eng Math 68:99-127

26. Dagher MA, Soldatos KP (2014) Pure azimuthal shear deformation of an incompressible tube reinforced by radial fibres resistant in bending. IMA J Appl Math 79:848-868

27. Dagher MA, Soldatos KP (2015) Area-preserving azimuthal shear deformation of an incompressible tube reinforced by radial fibres. J Eng Math 95:101-119

28. Dorfmann A, Merodio J, Ogden RW (2010) Non-smooth solutions in the azimuthal shear of an anisotropic nonlinearly elastic material. J Eng Math 68:27-36

29. Soldatos KP (2015) Modelling framework for mass-growth II: the general case. Mech Res Commun 65:35-42

30. Spencer AJM (1972) Deformations of fibre-reinforced materials. Clarendon Press, Oxford

31. Spencer AJM (1980) Continuum mechanics. Dover Publ, New York

32. Soldatos KP (2015) Small strain growth and the human nail. J Elast 124:57-80

33. Lubarda VA, Hoger A (2004) On the mechanics of solids with growing mass. Int J Solids Struct 39:4627-4664 